\title{
Long Extratropical Planetary Wave Propagation in the Presence of Slowly Varying Mean Flow and Bottom Topography. Part II: Ray Propagation and Comparison with Observations
}

\author{
Peter D. Killworth And JefFrey R. Blundell \\ Southampton Oceanography Centre, Empress Dock, Southampton, United Kingdom
}

(Manuscript received 15 November 2001, in final form 3 October 2002)

\begin{abstract}
Ray theory is used to predict phase and group velocities for long planetary waves under realistic, albeit slowly varying, oceanic conditions. The results are compared with local theory using fields smoothed to the same amount $\left(9^{\circ}\right.$ latitude/longitude) as well as those with much less smoothing $\left(1^{\circ}\right)$. The agreement is excellent, showing that local theory forms a good proxy for ray theory results. The predicted speeds agree well with observations of planetary waves deduced from sea surface height data. The theory uses purely baroclinic mean flow; the inclusion of barotropic flow has little effect except at high latitudes.
\end{abstract}

\section{Introduction}

In the first part of this paper (Killworth and Blundell 2003 , henceforth KBI), a generalized approach was given to describe how long midlatitude planetary waves propagate within a background mean flow, stratification, and ocean topography that are slowly varying compared with the length scale of the wave [a Wentzel-KramersBrillouin-Jeffreys (WKBJ) approach]. The solutions involved finding a dispersion relation which not only depended upon spatial position, but also on the horizontal orientation of the wavevector. ${ }^{1}$ This presented two difficulties. First, the actual orientation of the wavevector at any location depends presumably upon the details of the generation mechanism that produced the waves. Second, presentation of group velocities, etc., as functions of three variables (longitude, latitude, and wavevector orientation) is not straightforward. KBI avoided the second of these difficulties by noting that in much of the ocean the dependence of group velocity on wavevector orientation was weak, and they presented solutions which were averages over a wide range of westward wavevector orientations.

The first problem remains: how relevant are group velocities averaged over wavenumber orientations? For any specific forcing mechanism, the use of ray theory

\footnotetext{
${ }^{1}$ The dependence upon wave vector magnitude is purely linear for long waves and has no significant effect on calculations.

Corresponding author address: Dr. Peter Killworth, Process Modelling, James Rennell Division, Southampton Oceanography Centre, Empress Dock, Southampton SO14 3ZH, United Kingdom. E-mail: p.killworth@soc.soton.ac.uk
}

will provide a recipe for determining wavevector orientation at locations remote from the source of the waves. Killworth and Blundell (1999, hereinafter KB99) and Liu (1999) made respective use of this for waves propagating across topography, generated at an eastern boundary, and for waves forced by wind stress curl. Any ray problem requires knowledge of the dispersion relation (since rays move with the local group velocity). In KBI it was shown how to derive first derivatives of frequency with respect to both position and wavevector components given the frequency itself.

In this paper we shall examine ray theory solutions to the classical problem of planetary waves generated at an eastern coastline, following KB99. In that paper, they showed that topographic slopes had an important local effect on wave propagation, but averaged across an ocean basin there was little overall effect. Thus the speedup of planetary waves over their vertical normal mode solutions, observed by Chelton and Schlax (1996), could not be attributed solely to topographic slope effects, and, as noted in KBI, the modification of background potential vorticity by mean flow remains the most plausible candidate for speed increases. Indeed, KBI showed that the inclusion of both mean flow and topographic slope appears to yield a second speed increase over flat-bottom linear normal mode theory. However, this speedup depended crucially on the existing slope orientation; other (unrealistic) orientations could reduce the wave speeds. It was not clear why a fairly uniform speedup should then occur. Also following KB99, we shall compute the distribution of the surface elevation of the waves, since this is a quantity readily observable from satellite data. 
Section 2 gives a brief resume of the formulation, and section 3 sets up the ray theory equations. These are solved in each of the major basins in section 4, compared with the (averaged) local solutions in KBI in section 5 , and found to be highly similar. Section 6 compares the predicted (local) velocities with those deduced from observations. The effects of including a mean barotropic flow are briefly discussed in section 7 .

\section{Formulation}

The detailed formulation is given in $\mathrm{KBI}$, and only a brief overview is provided here. The planetary geostrophic approximation is made (restricting attention to long waves). Using Welander's (1959) $M$ variable, and denoting (temporal) mean values by overbars, and small perturbations by primes, we posit a local wavelike (WKBJ) solution

$$
M^{\prime}=F(\lambda, \theta, z) \operatorname{expi}(k \lambda+l \theta-\omega t),
$$

where $(\lambda, \theta, z)$ are respectively longitude, latitude and height, $(k, l)$ are the two components of a wavenumber, and $\omega$ is the wave frequency. The vertical structure $F$ is assumed to vary slowly laterally (i.e., on the basin scale) while the phase varies on the perturbation scale. This yields the self-adjoint eigenvalue system

$$
\left(\frac{F_{z}}{R}\right)_{z}+\frac{S}{R^{2}} F \equiv L(F)=0,
$$

where

$$
\begin{aligned}
S(z ; \lambda, \theta, k) & =\frac{k N^{2}(\lambda, \theta, z)}{a^{2} f \sin \theta} \\
Q(z ; \lambda, \theta, k, l) & =\frac{k \bar{u}}{a \cos \theta}+\frac{l \bar{v}}{a} \\
R(z ; \lambda, \theta, k, l) & =Q-\omega .
\end{aligned}
$$

This has boundary conditions

$$
\begin{aligned}
F & =0, \quad F_{z}=1, \\
z & =0 \text { (assuming a rigid lid) } \\
F(-H) & =-\alpha F_{z}(-H),
\end{aligned}
$$

where

$$
\alpha=\tan \theta\left(H_{\theta}-\frac{l}{k} H_{\lambda}\right) .
$$

Here $z=-H(\lambda, \theta)$ denotes the ocean floor, whose depth must also vary on the basin scale for WKBJ scaling to hold. (The second surface condition merely gives a scaling to the problem.)

If the frequency $\omega$ can be found, then phase velocities

$$
\mathbf{c}^{\mathrm{p}}=\left(c^{\mathrm{px}}, c^{\mathrm{py}}\right)=\left(\frac{\omega}{k} a \cos \theta, \frac{\omega}{l} a\right)
$$

(or, more properly)

$$
\left(\frac{\omega k}{k^{2}+l^{2}} a \cos \theta, \frac{\omega l}{k^{2}+l^{2}} a\right)
$$

and group velocities

$$
\mathbf{c}^{\mathrm{g}}=\left(c^{\mathrm{gx}}, c^{\mathrm{gy}}\right)=\left(\omega_{k} a \cos \theta, \omega_{l} a\right)
$$

can be computed. In the case of group velocities, this requires differentiating a dispersion relation which is essentially known only numerically. KBI show how this can be achieved, provided only that $\omega$ and $F$ are known at a horizontal location.

In order for ray theory to be used numerically, it is important that the location of caustics can be identified when they are reached (quite frequently, caustics display no obvious signature in terms of group velocity, for example). Caustics can be identified using the approach of KB99. This method involves being able to compute second derivatives of frequency with respect to all combinations of $\lambda, \theta, k$, and $l$. These too can be computed once $F, F_{X}, \omega$, and $\omega_{X}$ are known (using the methods in $\mathrm{KBI}$ ), where $X$ is any of $\lambda, \theta, k$, or $l$. The algebra for this is given in appendix $\mathrm{A}$.

\section{Ray theory approach}

We think of the dispersion relation now as being of the form $\omega=\omega(\lambda, \theta, k, l)$. If we assume a timelike coordinate $s$ along each ray, starting from some initial location, then ray theory gives (Lighthill 1978)

$$
\begin{aligned}
& \frac{D \lambda}{D s}=\frac{\partial \omega}{\partial k} \quad \frac{D \theta}{D s}=\frac{\partial \omega}{\partial l} \quad \frac{D k}{D s}=-\frac{\partial \omega}{\partial \lambda} \\
& \frac{D l}{D s}=-\frac{\partial \omega}{\partial \theta} .
\end{aligned}
$$

Rays move at the group velocity, modifying their wavevector as the ray propagates into regions with differing environments (in our case, different background mean flows, stratification, bottom depth, and bottom slope). The frequency is constant along each ray.

The equations (3.1) are solved numerically using routines from the NAG library (Numerical Algorithms Group 1999). All environment variables were computed as in KBI, using World Ocean Atlas data (Antonov et al. 1998; Boyer et al. 1998) and the ETOPO5 dataset (National Geophysical Data Center 1988) for topography, but with a $9^{\circ}$ Lanczos smoothing. This broad smoothing was used to avoid small-scale difficulties in the ray integrations, and results will be compared with 
local results with 1 and $9^{\circ}$ smoothing in section $5 .^{2}$ At each location, the vertical problem was solved using the method in KBI, but with 243 grid points in the vertical, this giving marginally fuller results.

The locations of caustics are found as follows, adapted from the approach of KB99. In addition to the alongray coordinate $s$, we define a cross-ray coordinate (basically a ray label) $\tau$. In the example given here, $\tau$ is distance along an eastern boundary, but could also be related to time of initiation of a ray, for example. Then since a caustic appears when two neighboring ray trajectories reach the same location sequentially (i.e., one at pseudotime $s$, and one at $s+\delta s$ ). This implies

$$
\begin{aligned}
& \lambda(s, \tau)=\lambda(s+\delta s, \tau+\delta \tau) \\
& \theta(s, \tau)=\theta(s+\delta s, \tau+\delta \tau) .
\end{aligned}
$$

KB99 show that this implies a caustic only when the Jacobian

$$
\frac{\partial \lambda}{\partial s} \frac{\partial \theta}{\partial \tau}-\frac{\partial \theta}{\partial s} \frac{\partial \lambda}{\partial \tau}
$$

becomes zero. While $\partial \lambda / \partial s, \partial \theta / \partial s$ are predicted from (3.1), the other two quantities involve a differentiation of (3.1). For example, differentiating the first of (3.1) gives

$$
\begin{aligned}
\frac{D}{D s}\left(\frac{\partial \lambda}{\partial \tau}\right)= & \frac{\partial^{2} \omega}{\partial \lambda \partial k} \frac{\partial \lambda}{\partial \tau}+\frac{\partial^{2} \omega}{\partial \theta \partial k} \frac{\partial \theta}{\partial \tau}+\frac{\partial^{2} \omega}{\partial k^{2}} \frac{\partial k}{\partial \tau} \\
& +\frac{\partial^{2} \omega}{\partial l \partial k} \frac{\partial l}{\partial \tau},
\end{aligned}
$$

which are linear in the $\tau$ differentials. Thus providing $\lambda, \theta, k$, and $l$ are known at initial locations and their variation with $\tau$ is specified, the rays may be integrated up to any caustics or regions in which solutions vanish (e.g., if they become complex).

Initial values for the rays depend crucially on the way the planetary waves are produced. We assume here, following KB99, that the waves are the result of windinduced upwelling events along an eastern boundary. This boundary is located at $\lambda=\lambda_{e}(\theta)$, which is a singlevalued function for the heavy smoothing used here. This location is operationally defined as being a contour of constant depth, taken here as $3500 \mathrm{~m}$. (We wish to avoid shallow water where both the slowly varying assumption and, indeed, much of the solution assumptions in general would break down.) The boundary lies at an angle $\tan ^{-1}\left(-H_{\lambda} / H_{\theta}\right)$ to the east since $H$ is constant along the boundary. If there is no normal flow into this

\footnotetext{
${ }^{2}$ The requirement that the mean states be horizontally smooth and that the mean flow be baroclinic are not easy to satisfy simultaneously if bottom topography varies. We chose first to make the mean flow baroclinic and then to smooth horizontally on the $9^{\circ}$ scale. The resulting mean barotropic flow is everywhere tiny (under $0.25 \mathrm{~mm} \mathrm{~s}^{-1}$ ) except near western boundaries and Drake Passage, areas which are largely out of consideration in what follows.
}

boundary, the wavenumber does not change along the boundary, and

$$
H_{\theta}-\frac{1}{k} H_{\lambda}=0, \quad \lambda=\lambda_{e}
$$

This implies, from (2.7), that $\alpha=0$ at the eastern boundary so that the ocean-as far as the dispersion relation is concerned-is essentially flat there. This means that the local problem may be solved with a specified orientation of the wavevector given by (3.5). Since the amplitude of the frequency merely scales with the wavenumber for long waves (cf. KBI), the eigenvalue problem is effectively free of parameters and can be solved locally. In practice we specify the frequency as annual and solve for $k$. Equation (3.5) then gives $l$ and, since $\lambda, \theta$ are already known, the system can be integrated forward.

In KB99 it was straightforward to follow energy density and the surface signature of the waves along the rays, using known formulas for energy density (entirely potential for long waves). In the presence of mean flow, the relevant quantity becomes wave action. Since this involves a division by the local intrinsic frequency (i.e., Doppler shifted by the mean flow), there are difficulties since we seek a two-dimensional ray propagation-unlike, say, Yang (2000) - yet the mean velocity varies with depth. ${ }^{3}$ The solution is to return to first (WKBJ) principles, detailed in appendix B. There it is shown that, if the waves have an amplitude $a_{0}(\lambda, \theta, k, l)$, taken to be uniformly unity on the eastern boundary, their evolution along a ray (ignoring all other effects such as local forcing, though these may well be important) is given by

$$
\frac{D a_{0}}{D s}=\sigma(\lambda, \theta) a_{0},
$$

where $\sigma(\lambda, \theta)$ is a complicated function of the local eigenfunction $F$ and some of its derivatives, resembling a horizontal divergence in form (as indeed it would reduce to in the absence of mean flow). Since $F$ has units of length from the surface normalization and $a_{0} F$ must have units of $L^{3} T^{-2}, a_{0}$ has units $L^{2} T^{-2}$; furthermore, as $F_{z}(0)=1, a_{0}$ is precisely the surface pressure perturbation.

\section{Solutions in realistic ocean basins}

Figures 1-5 show ray trajectories for the five major ocean basins. These share several characteristics. There is a tendency for a poleward turning of the trajectories soon after leaving the eastern sidewall. KB99 gave an argument for why this should be the case where there is no mean flow, but it is less clear why this should occur in the present case. The trajectories move

\footnotetext{
${ }^{3}$ We are indebted to a referee for pointing out that our first attempt at surface amplitude evolution had ignored the presence of mean flow.
} 


\section{N. Pacific rays}

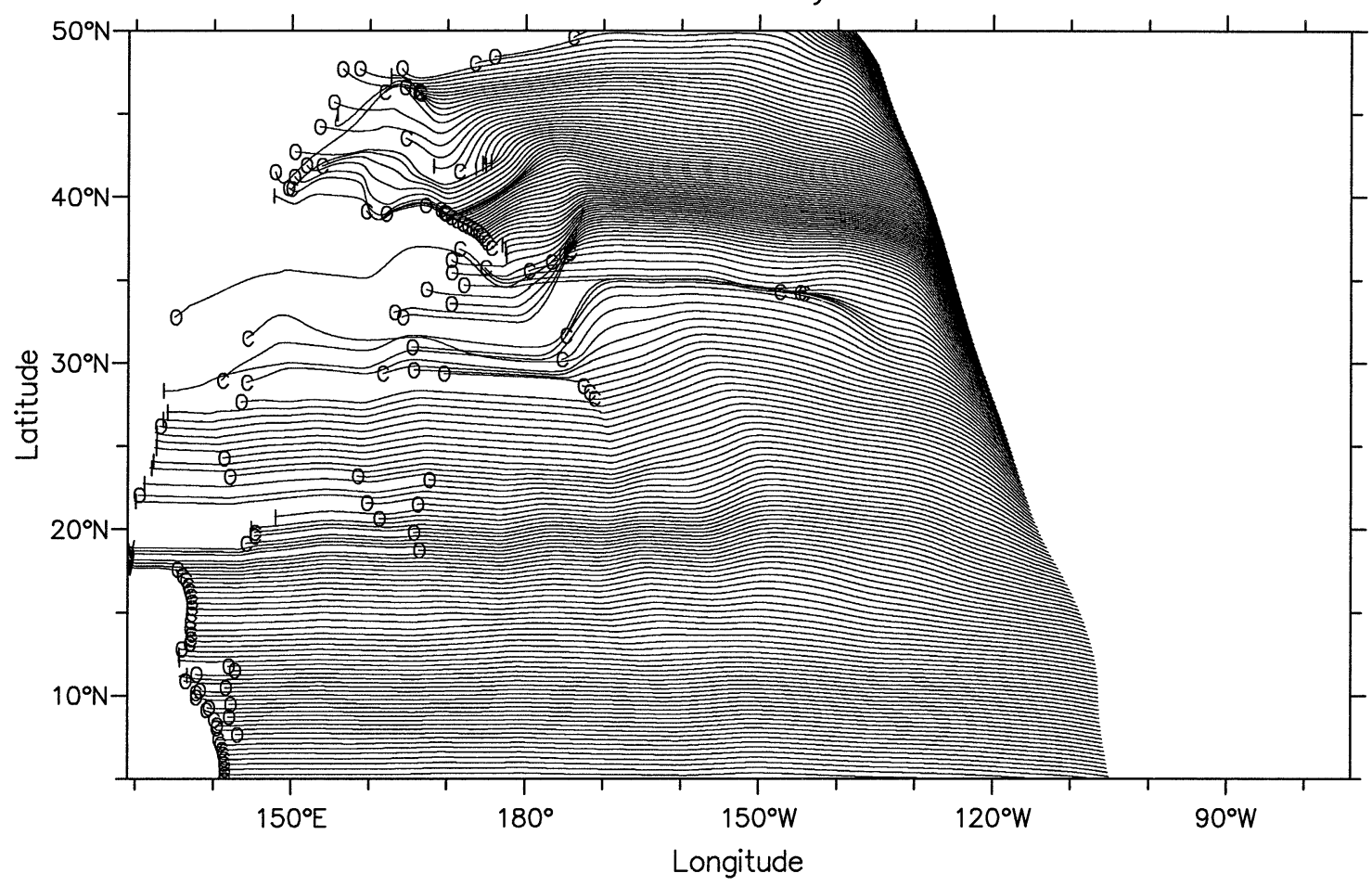

FIG. 1. Ray theory results for the North Pacific, showing trajectories, using an initial spacing between rays of $14^{\circ}$ : C marks caustics; D marks regions where rays enter depths less than $1000 \mathrm{~m}$, beyond which a ray is not followed; I marks regions where the integration routine failed; and $\mathrm{O}$ marks regions beyond which the bottom boundary condition (and, implicitly, frequency conservation) was not satisfied sufficiently accurately. A closer spacing of $1 / 8^{\circ}$ was used to compute the data for all diagrams without rays.

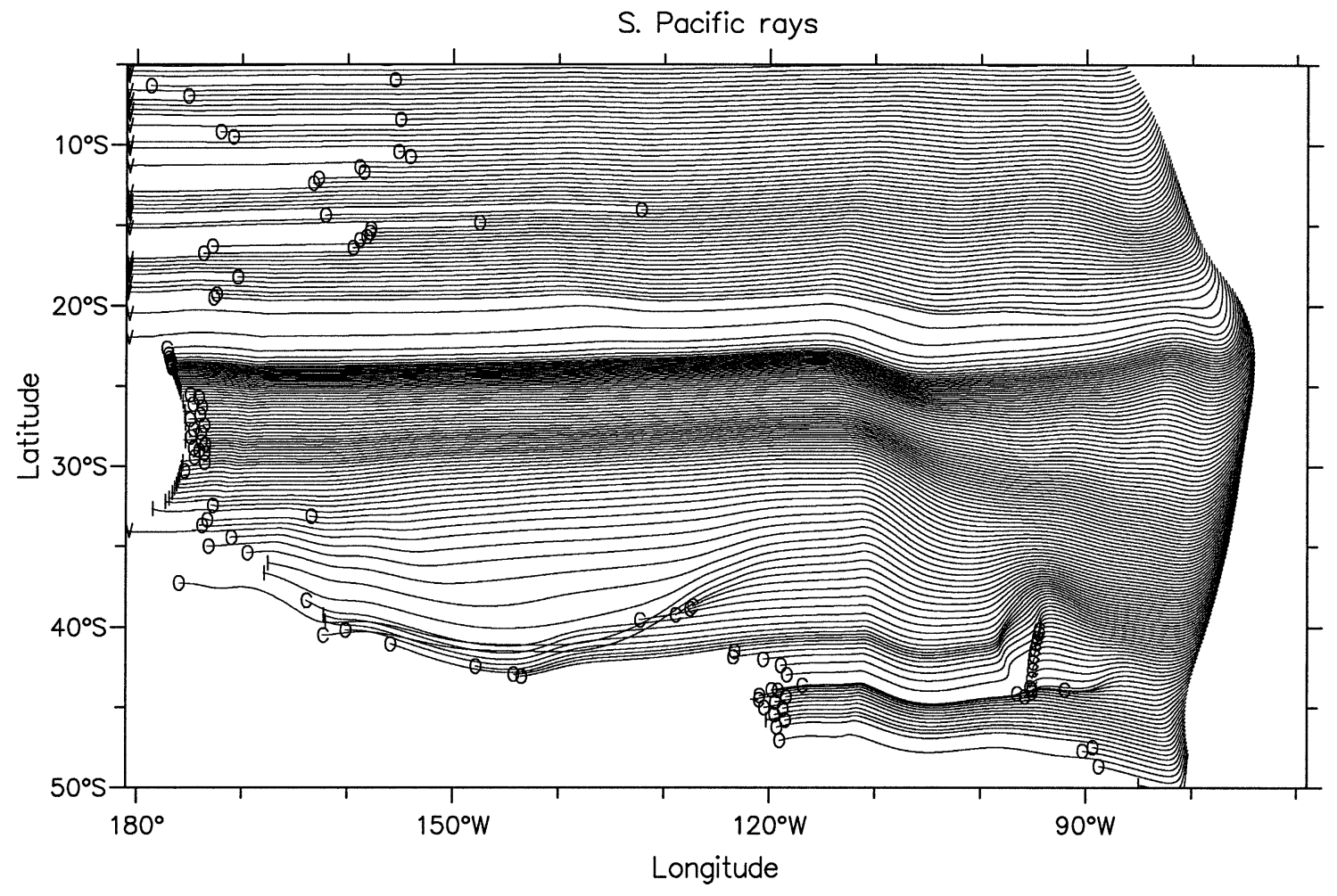

FIG. 2. Ray theory results for the South Pacific. Details as for Fig. 1. 


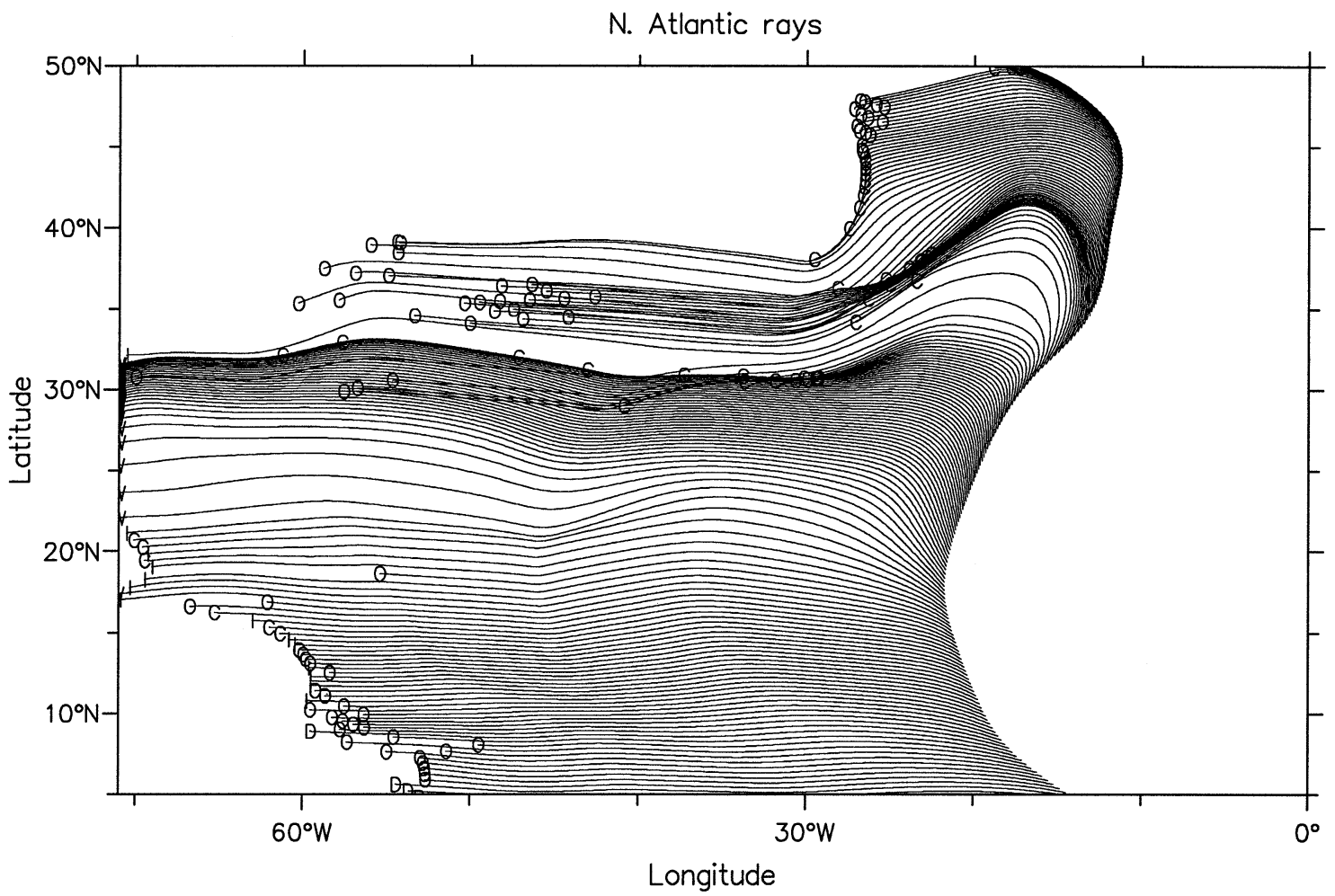

FIG. 3. Ray theory results for the North Atlantic. Details as for Fig. 1.

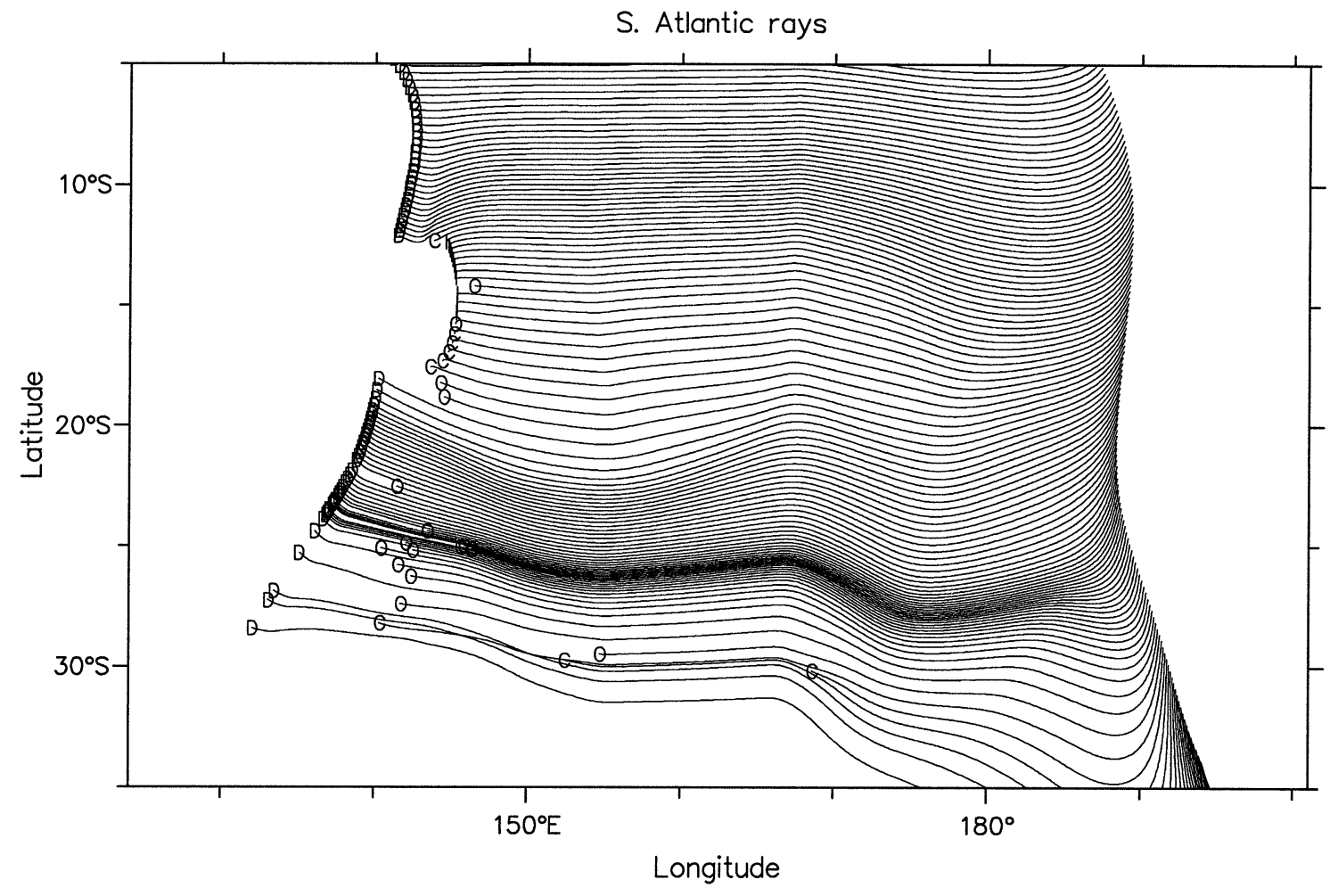

FIG. 4. Ray theory results for the South Atlantic. Details as for Fig. 1. 


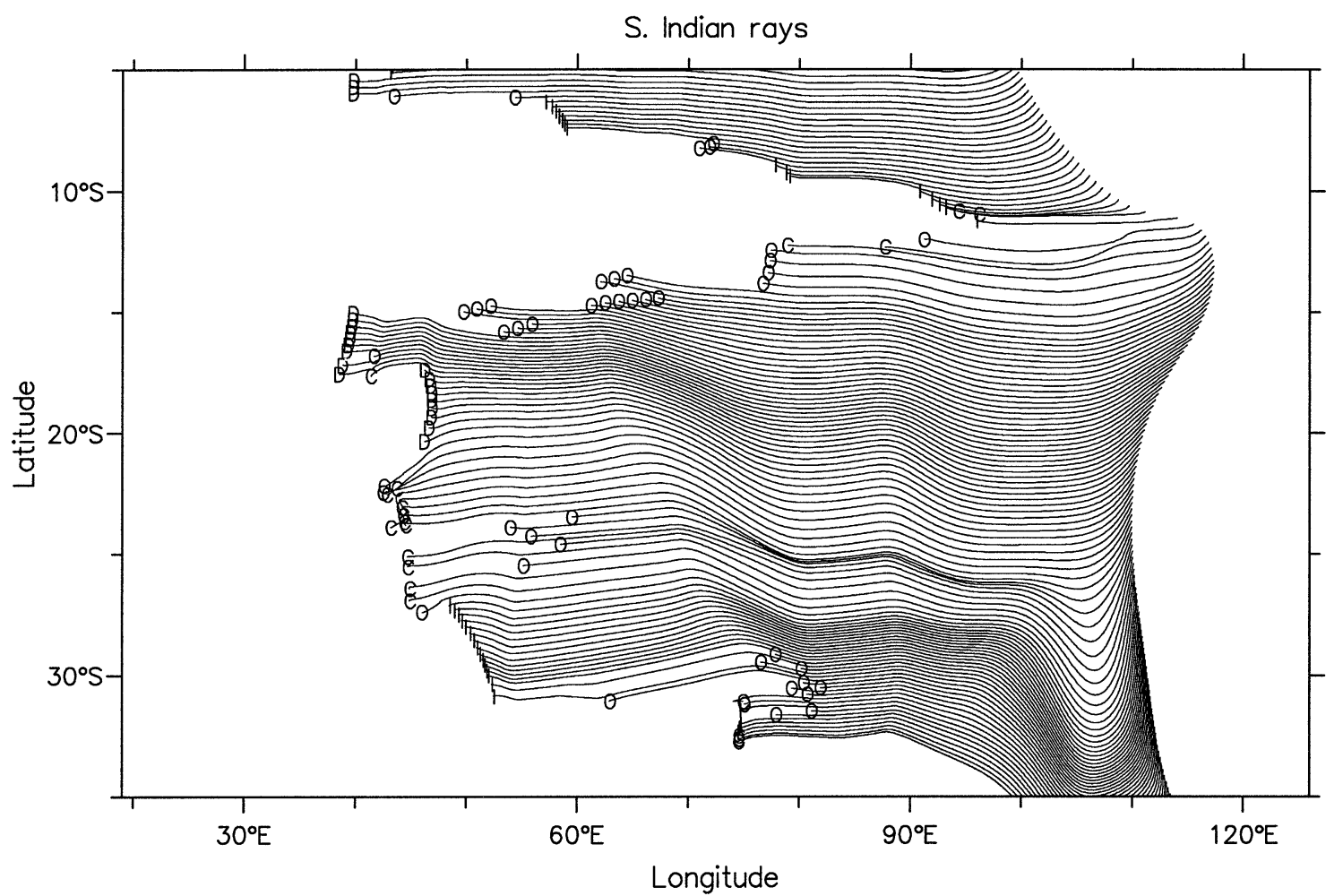

FIG. 5. Ray theory results for the South Indian. Details as for Fig. 1.

predominantly westward, reflecting the small northsouth group velocity (which is everywhere smaller than $1 \mathrm{~cm} \mathrm{~s}^{-1}$ except near the Mid-Atlantic Ridge and eastern boundaries). Rays initiated at high latitudes move equatorward so that there are few trajectories poleward of about $35^{\circ}-40^{\circ}$ in either hemisphere.

The striking fact about Figs. $1-5$ is their similarity to the trajectories of KB99 when there was no mean flow. Trajectories tend to terminate earlier than when there is no mean flow ${ }^{4}$ (mainly due to one of our test criteria involving monitoring accumulated bottom condition errors), but the patterns of convergences and divergences are almost identical to the earlier calculations. Convergences are seen in the North Pacific at $34^{\circ} \mathrm{N}$, South Pacific at $23^{\circ} \mathrm{S}$ and weakly at $43^{\circ} \mathrm{S}$, North Atlantic at $32^{\circ}-36^{\circ} \mathrm{N}$, South Atlantic at $25^{\circ} \mathrm{S}$, and South Indian weakly at $26^{\circ} \mathrm{S}$. Thus the bottom topographic slope appears to steer the rays almost independently of the mean flow. (Only group velocity direction is immediately visible from Figs. 1-5, of course, so group speed must be examined separately.)

Caustics appear in small regions in all basins, though the predominant cause for termination is the accuracy

\footnotetext{
${ }^{4}$ A measure of the error in the bottom boundary condition, and its cumulative value, is monitored along each ray. Were our numerics completely accurate, the bottom condition would be satisfied everywhere (i.e., $\omega$ would be the solution to each local problem). Errors tend to compound along rays, and, when they reach a cutoff value, we choose to terminate the ray.
}

of the bottom condition. Continuation methods across caustics do not seem to be available for the baroclinic cases here. In the North Pacific, there is a complex region of caustics north of $30^{\circ}-35^{\circ} \mathrm{N}$, while in the South Atlantic some caustics are visible south of $35^{\circ} \mathrm{S}$. Three strong caustics occur in the North Atlantic as part of the focusing effect: one removes propagation west of $30^{\circ} \mathrm{W}$ north of $40^{\circ} \mathrm{N}$; another is involved at $35^{\circ}-40^{\circ} \mathrm{N}$, $30^{\circ}-25^{\circ} \mathrm{W}$; and a third occurs oriented almost east-west at about $32^{\circ} \mathrm{N}$. The South Atlantic, by contrast, has only a weak caustic around $30^{\circ} \mathrm{S}$, whereas in the South Indian caustics terminate most rays between $22^{\circ}$ and $28^{\circ} \mathrm{S}$.

The solutions for separate basins were combined to form a truncated global dataset. Figures $6 \mathrm{a}$ and $6 \mathrm{~b}$ show the zonal phase and group velocity obtained by this process (the continental outlines give a feel for the topographic smoothing which has occurred). The two fields are visually similar, both being predominantly zonally symmetric, but differ in detail and in magnitude. These differences are shown in Figs. 7 and 8, which display the zonally averaged speeds and their ratio. In the Northern Hemisphere, the fall-off of speed with distance poleward is more gradual than in the local theory (compare with Fig. 6 in KBI). In the Southern Hemisphere, the differences between phase and group become pronounced south of around $40^{\circ} \mathrm{S}$, though as Figs. 6a and $6 \mathrm{~b}$ show, the number of points on which the averages are based becomes small at such latitudes. There is a tendency for phase velocity to exceed group velocity 
(a)

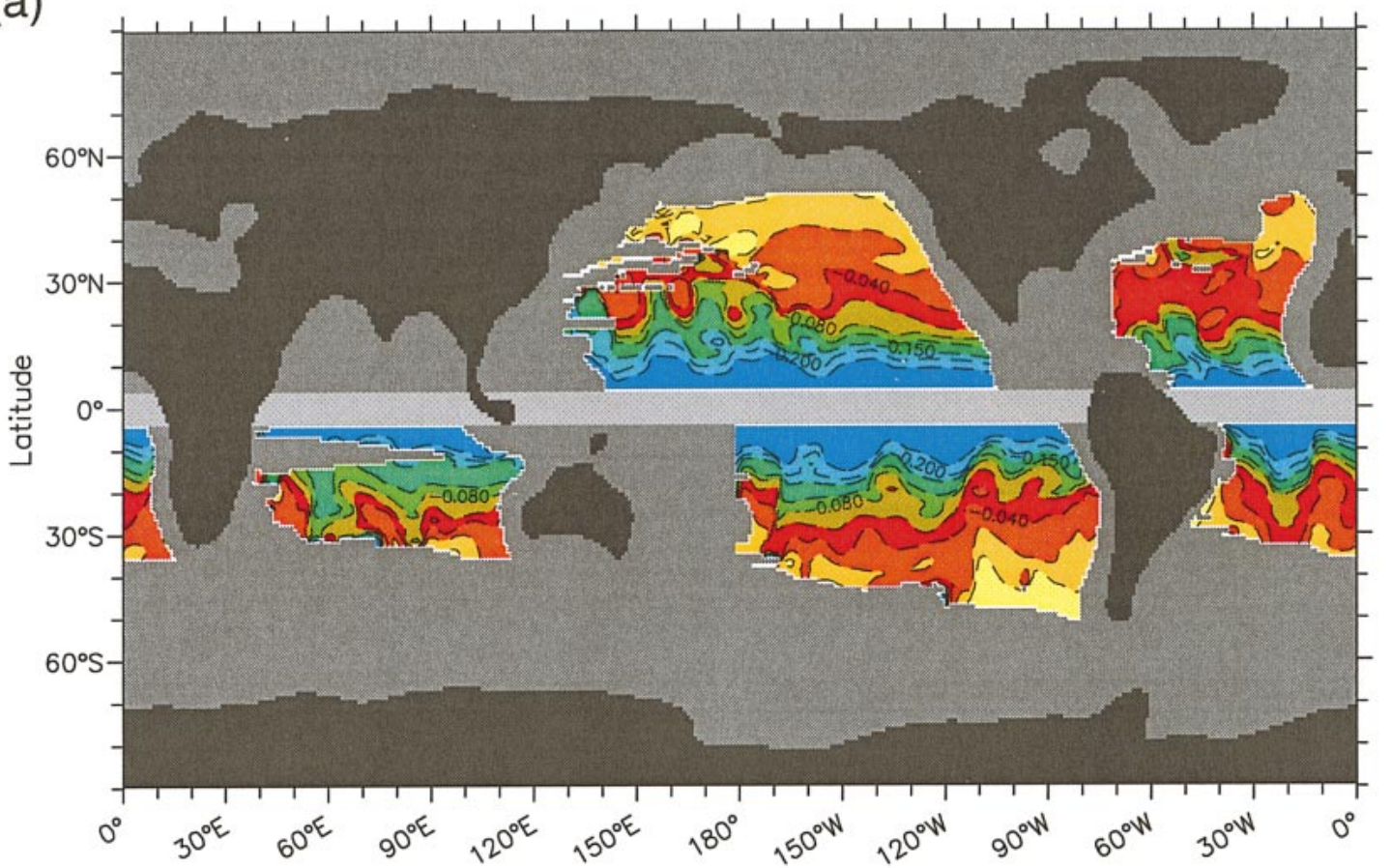

(b)

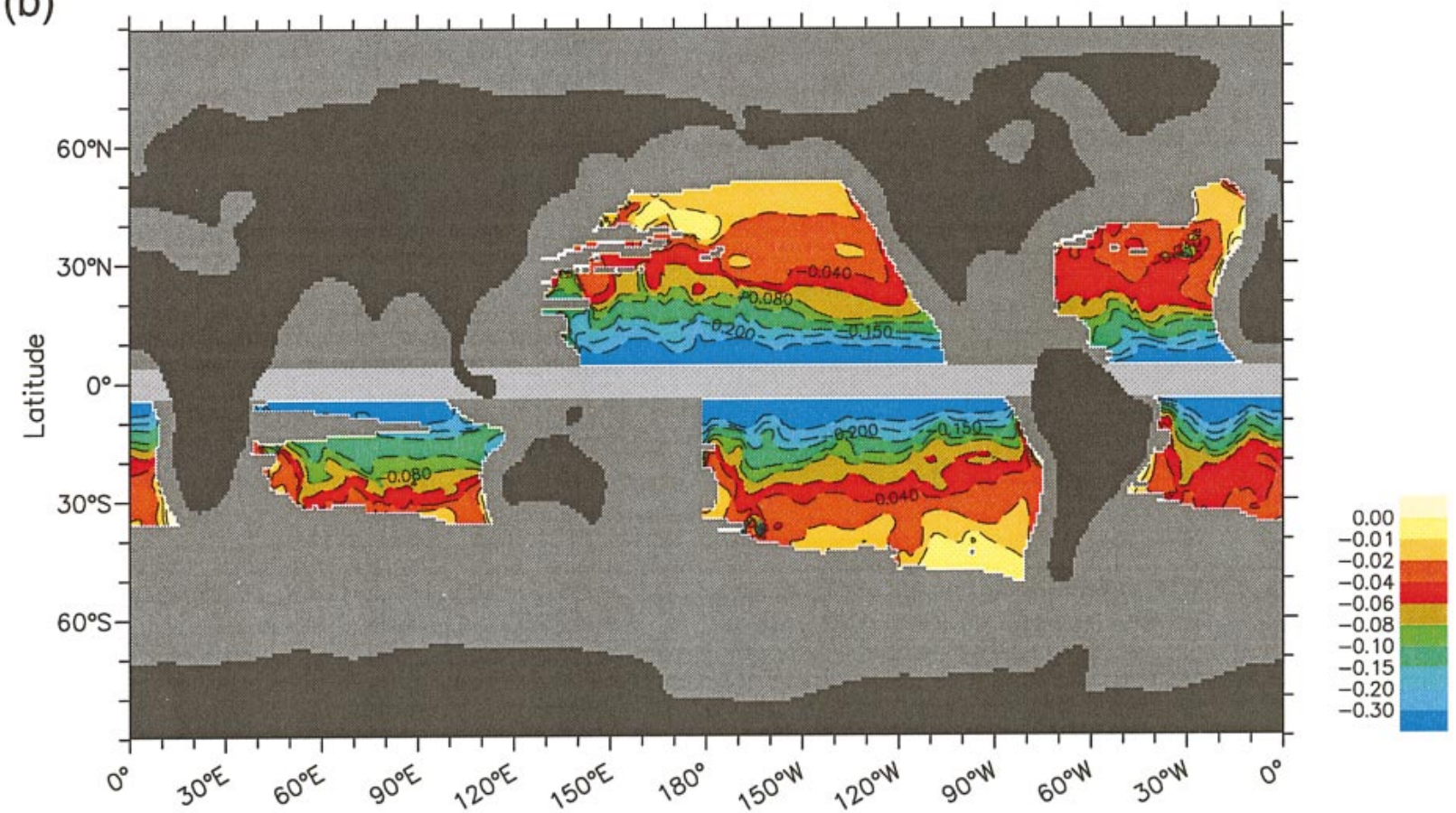

\section{Longitude}

FIG. 6. East-west velocities obtained from concatenation of the ray theory results in each ocean basin: (a) phase, (b) group. Contour interval is nonuniform $\left(0,-0.01,-0.02,-0.04,-0.06,-0.08,-0.10,-0.15,-0.20,-0.30 \mathrm{~m} \mathrm{~s}^{-1}\right.$ to display detail). Areas where ray theory could not reach are shaded dark gray. Near-equatorial regions are also not shown, since midlatitude theory does not hold there. 


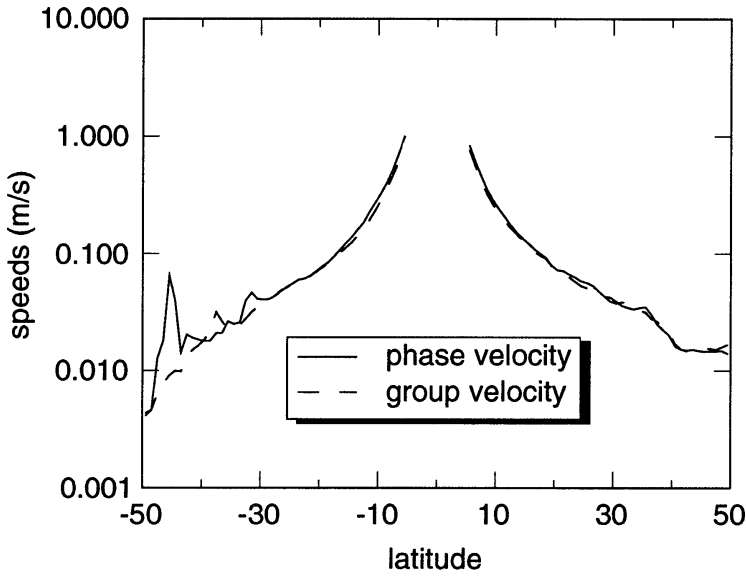

FIG. 7. Zonally averaged phase and group speeds from the ray theory results, as a function of latitude.

slightly in magnitude (Fig. 8) over much of the midlatitudes; we know of no rationale for this.

The wavevector (not shown) behaves predictably. The east-west component $(k)$ has a fairly zonal structure, running between values of order -10 at low latitudes to -30 or more at higher latitudes. [For a flat bottom and zonal flow, $k$ would not vary westward; cf. KBI's Eq. (3.9).] The north-south component, however, increases in magnitude westward [cf. Schopf et al. (1981) for a discussion], reaching values of 100-200 (which is into the range where long-wave theory is becoming less valid).

Figure 9 shows the surface elevation, on an arbitrary scale, predicted for rays initiating from the eastern boundary (recall that the elevation is taken as uniform along the eastern boundary, and that there is no other source of energy for the rays). Over most of the ocean the surface elevation varies between about 0.2 and 1.6, and for the moment we shall discuss only these areas. Consistently within each basin two features occur. Nearequatorial areas show an increase in surface elevation, and areas west of the midocean ridges show a decrease in surface elevation. A consistent region of larger amplitude occurs across the South Pacific at $24^{\circ} \mathrm{S}$; this feature was also found by KB99.

These findings do not agree well with the findings of $\mathrm{Fu}$ and Chelton (2001) at $24^{\circ}$ latitude, nor with amplitude maps for the Pacific (D. B. Chelton 2001, personal communication) or globally (P. Cipollini 2001, personal communication). Fu and Chelton found fairly uniform amplitudes across the basin in the South Atlantic, with a hint of increased amplitude at $40^{\circ} \mathrm{W}$, which does agree with our results. In the South Indian, they found considerably larger values uniformly across the basin; our area of large amplitude around $50^{\circ} \mathrm{E}$ is not observed. All estimates find amplitude increases west of midocean ridges, whereas our results predict the opposite. The exception is Cipollini's results for the South Pacific; he finds a broad band of larger amplitude across the entire

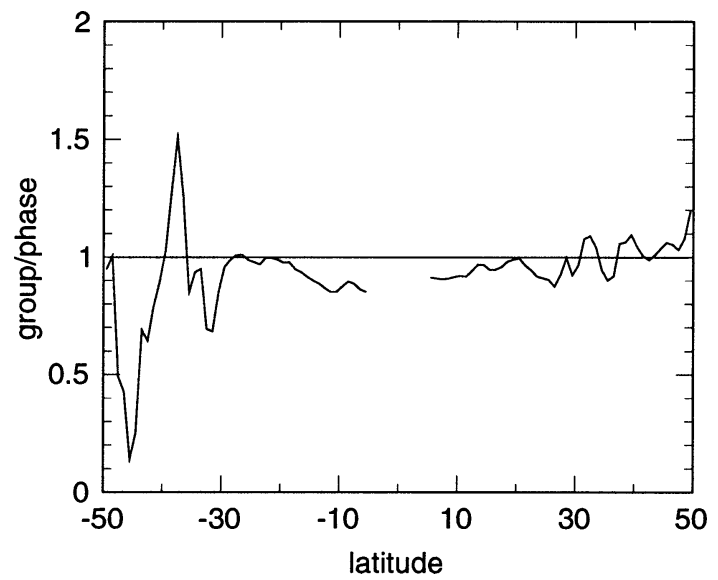

FIG. 8. The ratio of group to phase velocity, using the zonally averaged speeds from Fig. 7. The variability in southern latitudes partly derives from the small number of data points in such regions.

basin centered on $20^{\circ} \mathrm{S}$. Our results show a similar, but narrower, band at the same latitude. At the very least, though, the decrease in amplitude west of midocean ridges appears to be evidence that planetary waves are generated by more than a single mechanism, for example, by direct wind forcing, and especially by additional forcing by topographic ridge effects including possible mode-mode interactions. Examples of such effects are given by Tailleux and McWilliams (2000), Hallberg (1997), Barnier (1988), and Anderson and Killworth (1977). Note that all these papers use a two-layer approach which appears to overestimate the effects of topography. The unforced ray model neglects all these effects.

Each ocean basin possesses regions where the rhs of (3.6) behaves similarly to what would be a region of convergence of group velocity in the unsheared case. In such regions the surface elevation increases rapidly, often to values of many thousands, which is certainly unrealistic. The most noticeable regions for large increases in amplitude are west of $180^{\circ}$ between $38^{\circ}$ and $45^{\circ} \mathrm{N}$, and far west at $28^{\circ} \mathrm{N}$ in the North Pacific; $170^{\circ} \mathrm{W}$, $35^{\circ} \mathrm{S}$ and $115^{\circ} \mathrm{W}, 42^{\circ} \mathrm{S}$ in the South Pacific; between $5^{\circ}$ and $20^{\circ} \mathrm{S}$ in the South Indian west of $60^{\circ} \mathrm{E}$ (though numerical solution loss in this region makes it hard to be precise); a small area between $15^{\circ}$ and $25^{\circ} \mathrm{S}$ near the western boundary in the South Atlantic; and, most notably, north of $32^{\circ} \mathrm{N}$ in the North Atlantic. This last area is a popular one for the reliable recovery of planetary wave signals from remote observations (Cipollini et al. 1997). Many, but not all, of the areas of large elevation also appeared in the no-mean-flow case of KB99, ${ }^{5}$ who

\footnotetext{
5 The computations were repeated with the mean flow set to zero for more immediate comparison with $\mathrm{KB} 99$, as well as a check on the algebra. Subject to the proviso that $N^{2}$ now varies across the basin, and the use of a more recent global dataset, the results in this case compare well with KB99.
} 


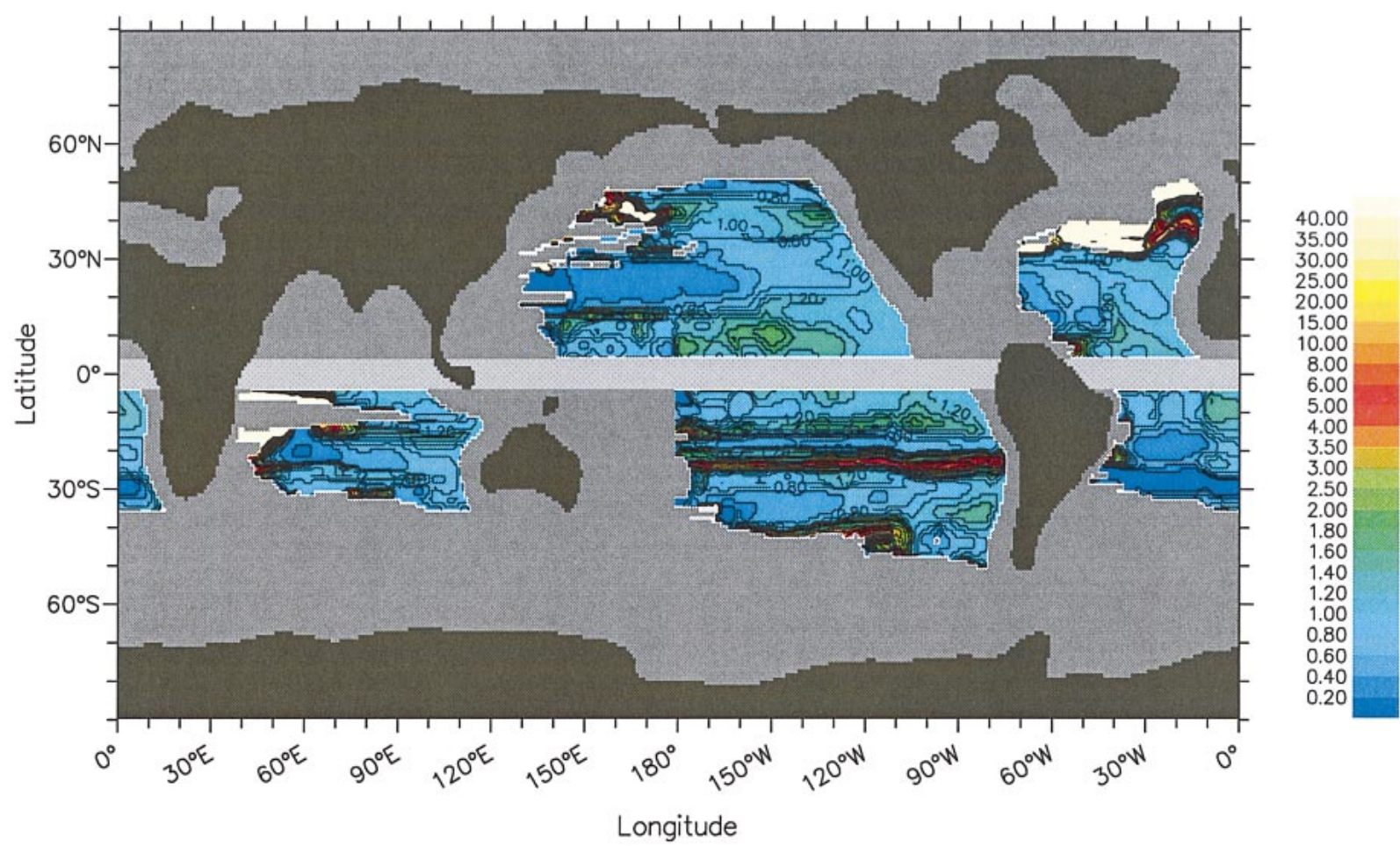

FIG. 9. The surface elevation predicted from ray theory (with an arbitrary but uniform amplitude along the eastern boundary). Because of the wide variation, contour intervals are nonconstant. They are $0.2-2$ by $0.2 ; 2.5-4$ by $0.5 ; 5,6$, and $8 ; 10-40$ by 5 .

also found focusing in sea surface height and temperature signals at the same North Atlantic location.

\section{Comparison with local solutions}

The results of the previous section cannot be compared directly with the results of KBI since their data used a $1^{\circ}$ smoother rather than the $9^{\circ}$ smoother used for the ray theory. Instead, the local calculation of KBI was first repeated using the $9^{\circ}$ smoothed data. Figure 10 shows contours of the east-west group velocity, locally computed using these data; the diagram may be compared visually with Fig. $4 \mathrm{a}$ in KBI, which used $1^{\circ}$ smoothed data. The two computations are clearly similar in all but detail. Numerical confirmation is given in Fig. 11, which shows a scatterplot of the local results for east-west group velocity using $9^{\circ}$ smoothing against those using $1^{\circ}$ smoothing, with a very few abnormal outliers removed (created when the group velocity calculation in KBI has a nearly zero denominator). While there is scatter, the correlation is 0.97 , though this is dominated by the large near-equatorial speeds that are less affected by the relatively low mean flows there (the diagram conceals many overplotted points lying on the diagonal). Nonetheless, the effects of smoothing are to lessen the effectiveness of the $9^{\circ}$ description: fully $25 \%$ of the $9^{\circ}$ results lie outside 1.96 standard deviations from the $1^{\circ}$ theory. Figure 12 shows the same data, but restricted to speeds under $0.03 \mathrm{~m} \mathrm{~s}^{-1}$, to concentrate on higher-latitude differences. Again, there is scatter (the correlation is 0.88 , with $22 \%$ of the data differing from the $1^{\circ}$ value by more than 1.96 standard deviations), but it is clear that the smoothing is not having a large effect on the net amplitude of the wave speed. As final confirmation of similarity, Fig. 13 shows the east-west group speed, averaged by latitude bands, for the $1^{\circ}$ and $9^{\circ}$ smoothed local calculations. Apart from a hint that the $9^{\circ}$ data underestimate the $1^{\circ}$ data slightly in the Southern Hemisphere, the two results are remarkably similar.

With this preamble we may now compare the ray theory results with local $9^{\circ}$ theory (Figs. 6b and 10, respectively). With some exceptions, the two fields are similar where they may be compared, though there is more hint of near-equatorial variability in the ray theory results. Figure 14 shows zonal averages of the local $9^{\circ}$ group speed compared with ray theory estimates. These are extremely similar except for areas south of about $35^{\circ} \mathrm{S}$, where ray theory is first faster and then slower than the mean $9^{\circ}$ values. The differences seem to be largely confined to certain areas (e.g., $33^{\circ} \mathrm{S}, 175^{\circ} \mathrm{W}$ ) in which the ray theory, by selecting certain orientations for the wavevector, has yielded group velocities at the lowest end of the range found by local theory. Note again the lesser fall-off of the predicted speeds in the Northern Hemisphere with distance poleward than is observed using $1^{\circ}$ smoothing (cf. KBI, Fig. 6); this may 


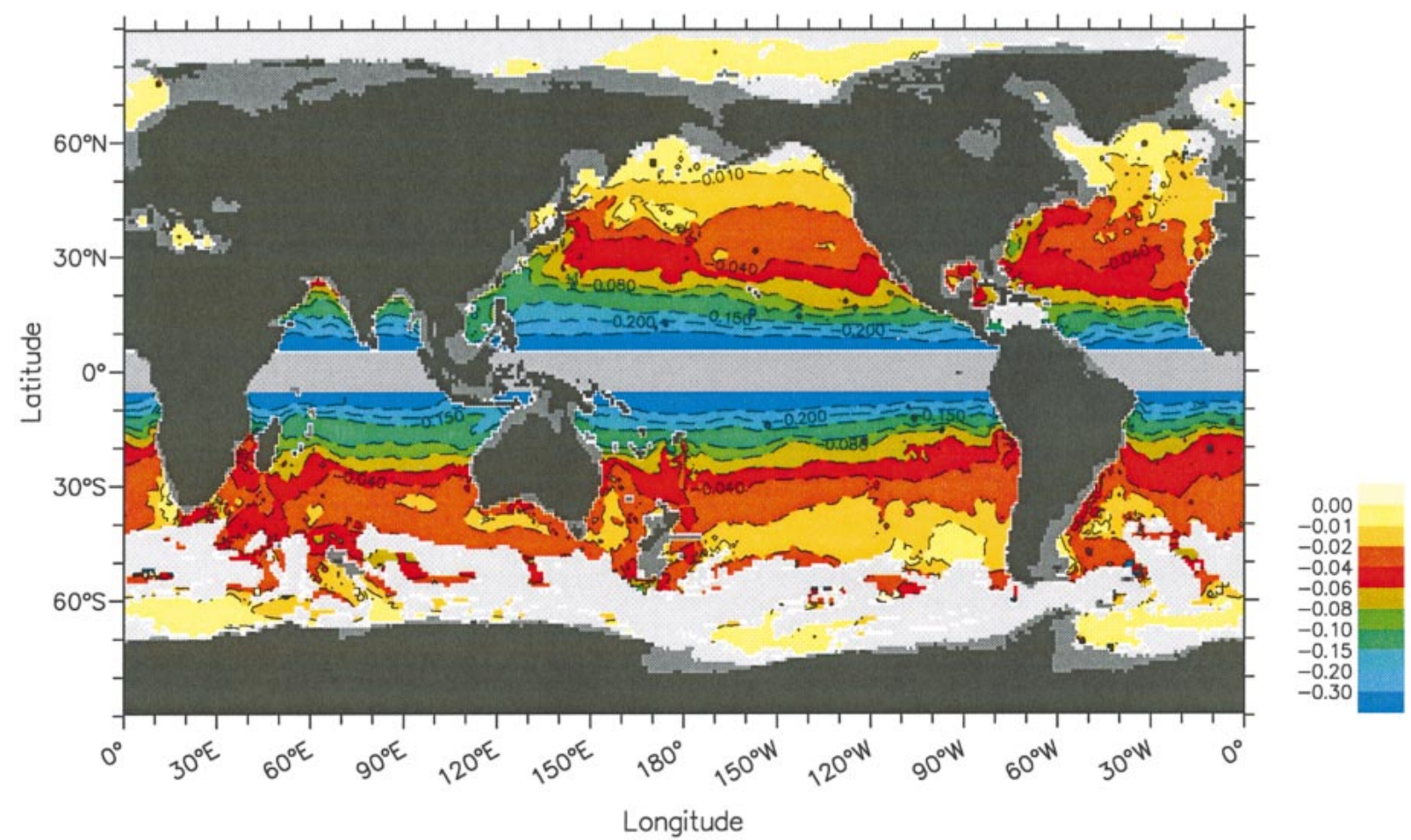

FIG. 10. East-west group velocity obtained from local theory using data smoothed on a $9^{\circ}$ scale, for comparison with Fig. $4 \mathrm{a}$ of KBI. Contour interval is nonuniform $\left(0,-0.01,-0.02,-0.04,-0.06,-0.08,-0.10,-0.15,-0.20,-0.30 \mathrm{~m} \mathrm{~s}^{-1}\right.$ to display detail). Nearequatorial regions are not shown since midlatitude theory does not hold there.

partly be produced by the differing longitudes at which solutions can be found.

Thus, in general, the results of ray theory do not appear to show any significant bias from the local theory presented in KBI. In particular, there appears to be little if any tendency for a ray initially created as mode 1 to

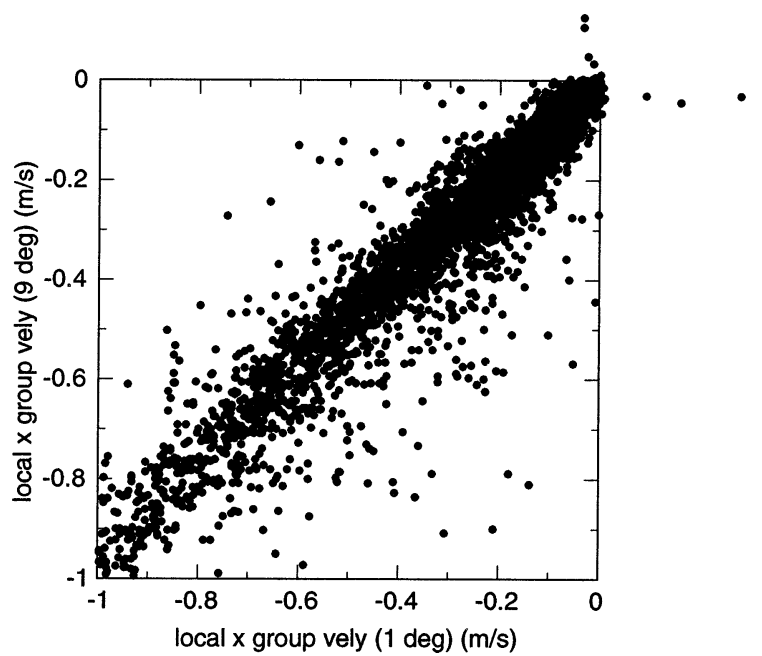

FIG. 11. Scatterplot of east-west group velocities obtained from $1^{\circ}$ and $9^{\circ}$ smoothed local calculations, with a few outliers removed. be modified during its propagation into mode 2 (if there were, the comparison of contours of the ray group velocity and the local mode 1 group velocity would show large differences where the rays had been converted to mode 2).

\section{Comparison of local results with observations \\ a. Radon transform wave speed data}

The original observations by Chelton and Schlax (1996), and subsequently updated to include later data (Fu and Chelton 2001), were used as a benchmark of the success or failure of the mean-flow hypothesis of Killworth et al. (1997). In these observations, there are a set of latitude bands. For each band, an observed planetary wave speed between a specified western and eastern longitude is known, and can be compared with theory by computing the ratio of observed to theoretical wave speeds. This ratio, for the varying theories, is shown in Fig. 15. (The notation remains as in KBI. Each possibility is denoted by a pair of letters. The first is $\mathrm{N}$, $\mathrm{Z}$, or $\mathrm{G}$, referring to no mean flow, zonal mean flow, or general mean flow respectively. The second is F or $\mathrm{S}$, referring to flat bottom and sloping bottom, respectively.)

The NF and GF results, subject to small changes in datasets, essentially repeat Killworth et al. (1997). The 


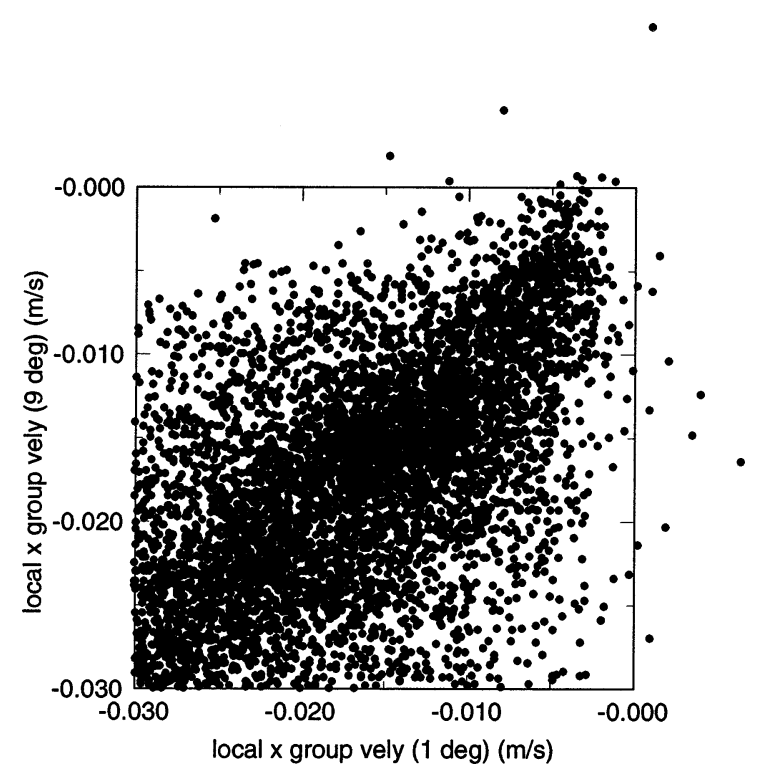

FIG. 12. As in Fig. 11 but limiting data to speeds under $0.03 \mathrm{~m} \mathrm{~s}^{-1}$.

NF results show the familiar large ratio indicating the failure of standard linear theory. The improvement in the $\mathrm{ZF}$ results is now well documented. As an example, compare with Fu and Chelton's (2001) comparisons of radon transform results with $\mathrm{ZF}$ theory. Their estimates of the latter differ slightly from ours for various numerical reasons: our use of slightly smoothed topography, the later World Ocean Atlas data, real solutions from integrating over approximately 126 grid points rather than a possibly complex matrix inversion over about 35 points, solution of the equation for pressure rather than for $M$, and so on. However, the ratio ( $\mathrm{ZF}$ speed/Fu and Chelton speed) has a mean of 0.99 but a standard deviation of 0.11 , indicating some variation between the two sets of results. ${ }^{6}$

The results from the GF theory show a strong reduction in the erroneously large ratios of standard linear normal modes (NF), though with some residual ratios which are still somewhat high, particularly in the Southern Hemisphere. Conversely, the NS results (inherent in KB99) show little net change in propagation speeds from the traditional NF results: a slope in one direction that speeds the propagation is usually followed by the opposite slope that acts to decrease the speed, resulting in little change in basin propagation times from NF theory.

The last panel in Fig. 15 demonstrates that the second speedup visible in the global results in the new GS case is also present when limited to the $\mathrm{Fu}$ and Chelton

\footnotetext{
${ }^{6}$ The difference is strongly reduced without the two southernmost points, at $46^{\circ}$ and $48^{\circ} \mathrm{S}$, where our ZF estimates are about 0.6 of the $\mathrm{Fu}$ and Chelton estimates; the mean of the ratio over the remaining points is 1.00 , std dev 0.08 .
}

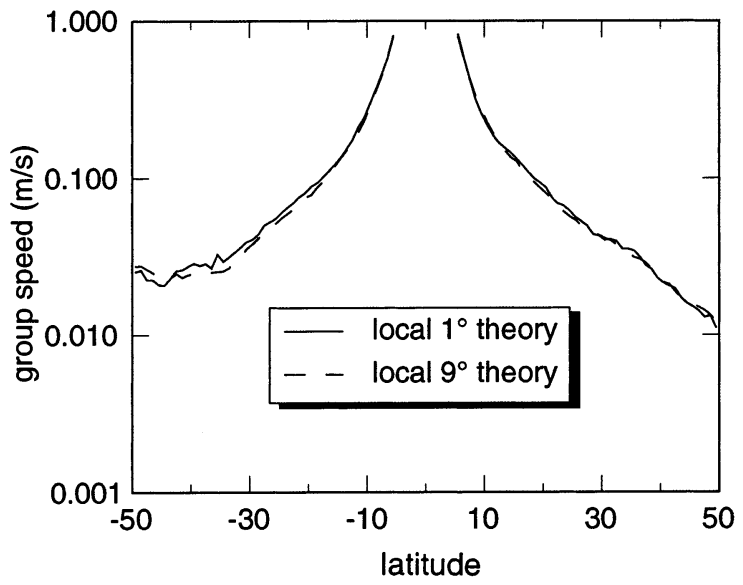

FIG. 13. East-west group speeds, averaged around latitude circles, for results from $1^{\circ}$ and $9^{\circ}$ smoothed local calculations. Note the logarithmic scale for the speeds.

(2001) longitude bands. The ratio of observed to GS speeds is now essentially unity in the Northern Hemisphere, and only slightly above unity in the Southern Hemisphere. Thus the GS theory appears to account for the vast majority of the observations of planetary wave speed in the ocean.

Alternative estimates of wave speeds, using different smoothing algorithms, can be made from the altimeter data. Cipollini (2001, personal communication) has recomputed observed speeds on the same longitude bands as Fu and Chelton (2001), also using a radon transform (cf. Hill et al. 2000 for details) and finds variations in the ratios of his results to those of $\mathrm{Fu}$ and Chelton to be of the same order as those in Fig. 15 comparing the GS theory to those data. This gives an estimate of how close data and theory might be expected to lie.

In addition, Cipollini has computed observed wave speeds, for each $1^{\circ}$ latitude band, across as wide a set

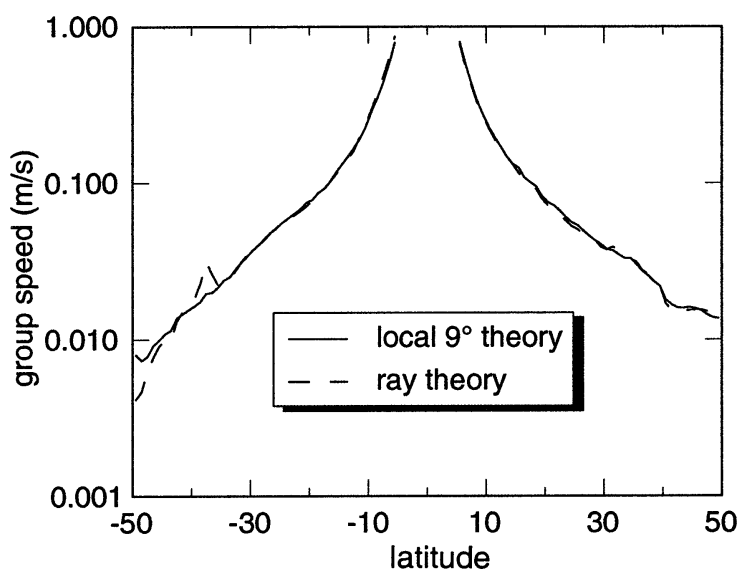

FIG. 14. Zonal averages of westward group speed for local $9^{\circ}$ theory and for ray theory, shown on a logarithmic scale as a function of latitude. 

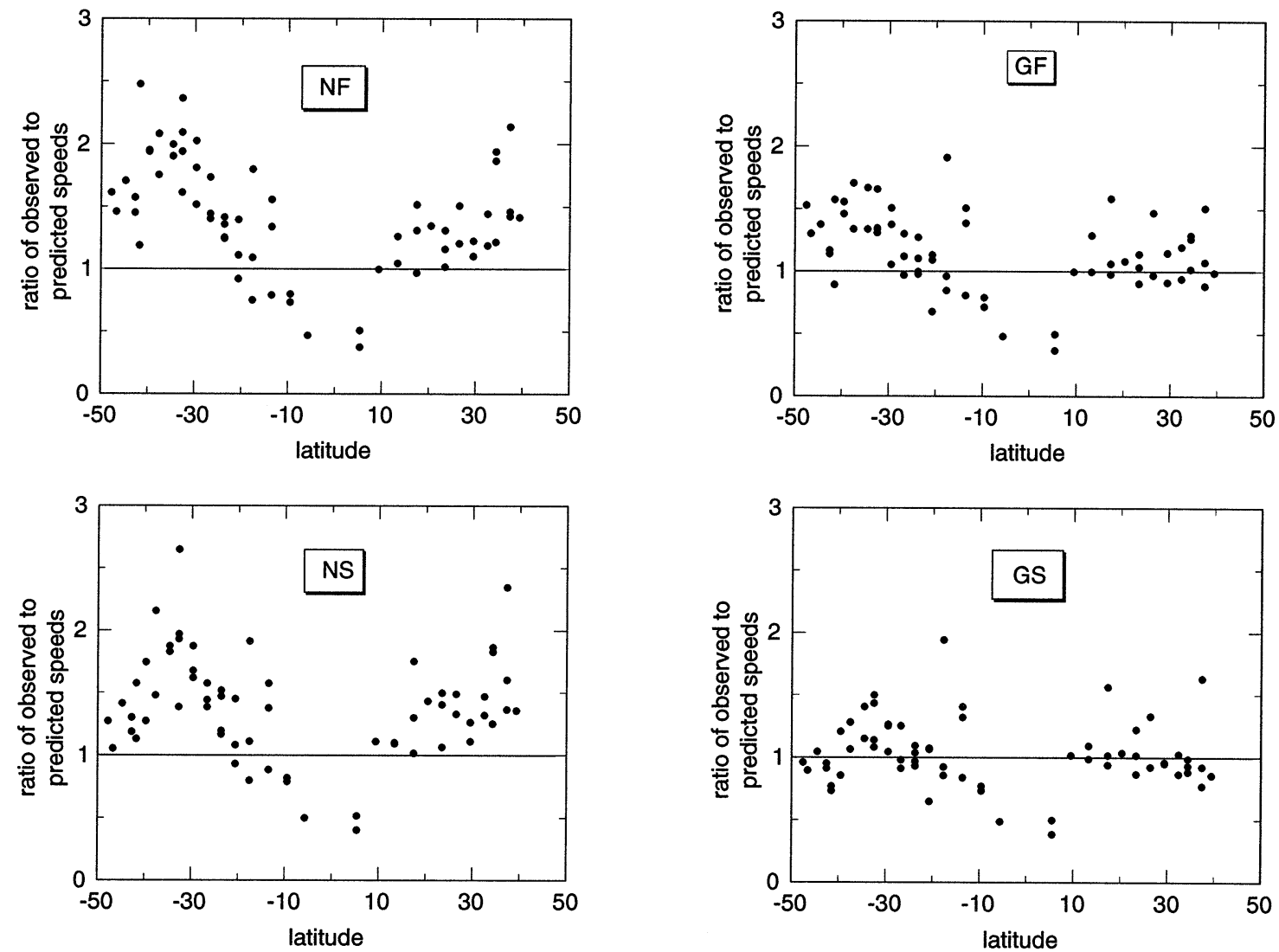

FIG. 15. The ratio of phase speeds observed by Chelton and Schlax (1996) to predicted westward group velocities. Each point represents an average over a longitudinal band (for clarity, the different ocean basins have not been distinguished). The $\mathrm{ZF}$ and ZS cases are omitted because they are indistinguishable from the respective GF, GS cases.

of ocean swaths as possible (thus providing up to three speed estimates per latitude band). The GS theory results were averaged on the same bands, and the results are shown in Fig. 16. The observations show some scat-

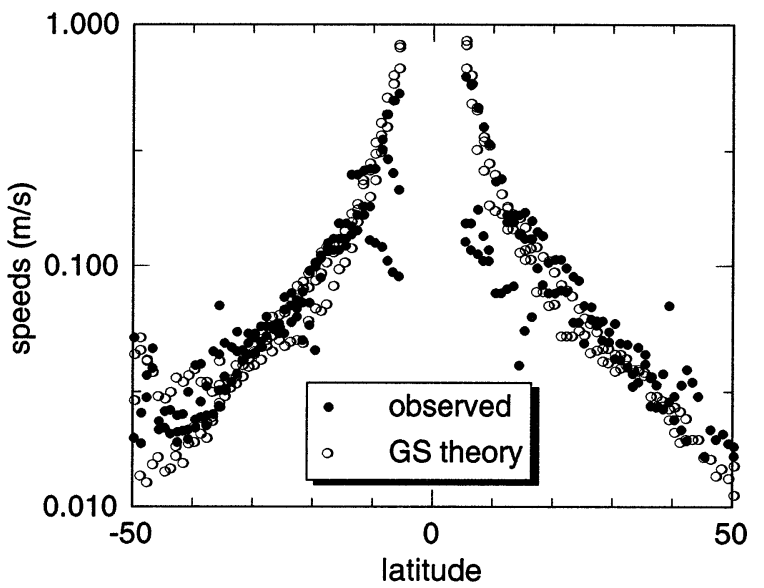

FIG. 16. Westward observed (data from P. Cipollini 2001, personal communication) and GS speeds, averaged on longitude bands spanning ocean basins. ter, particularly at lower latitudes, and there are clear differences between basins at high southern latitudes. The GS theory clearly does an excellent job of reproducing these observations, although somewhat low at very high southern latitudes, and predicts too fast wavespeeds at low latitudes. The latter is a common problem, as Fig. 15 also demonstrates; the observational analysis procedure may be selecting a higher internal mode due to the long wavelengths and high speeds involved at low latitudes (also discussed in the next subsection).

\section{b. White's near-equatorial data}

White (2000a,b), filtering the data to remove shorterperiod waves, observed longer-period waves in the equatorial Pacific Ocean (2000b) and southern equatorial Indian Ocean (2000a). These waves are much slower than those reported by $\mathrm{Fu}$ and Chelton (2001) for the same regions; indeed, $\mathrm{Fu}$ and Chelton found a westward speed of $0.28 \mathrm{~m} \mathrm{~s}^{-1}$ at $10^{\circ} \mathrm{S}$ in the Pacific, compared with White's 0.15 . White argued that his observations might not be those of oceanic planetary waves, even taking the Killworth et al. (1997) theory into account, because of the difference in speeds. Ac- 


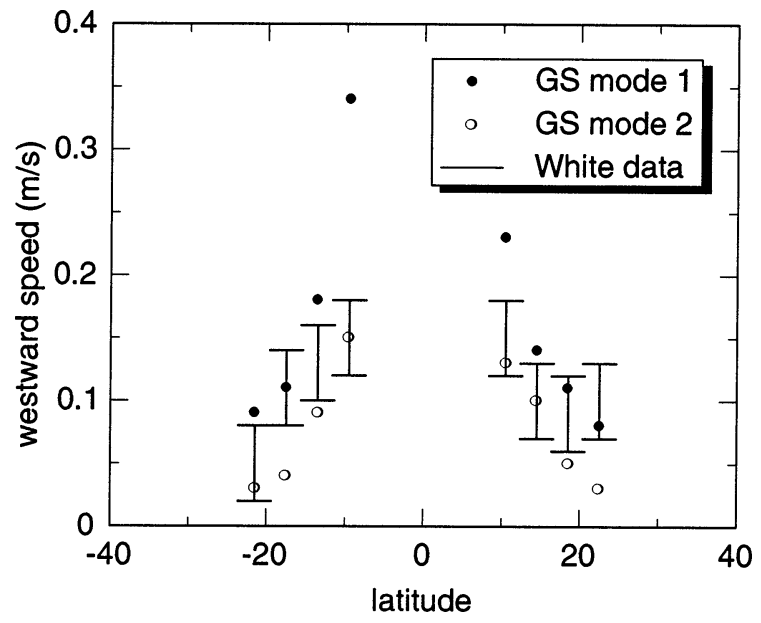

FIG. 17. Phase speeds for the lat-lon ranges in the equatorial Pacific used by White (2000b). The error bars show White's data (at the midpoint of the error); the filled circles show the first GS mode speed averaged over the same longitude band as White; and the open circles show the second GS mode speed.

cordingly, he created two different theories (one for the Pacific, one for the Indian) involving complex interactions with the atmospheric circulation, to deduce the properties of coupled planetary waves. While the signals he observes may well be coupled waves (White notes the co-varying meridional surface wind signal, for example), it does ignore the potentially much simpler possibility that the waves White observes are higher vertical modes of the oceanic system. We examine this possibility here for the Pacific Ocean, but only comment on the Indian Ocean where free modes do not seem to account for White's data [though free modes account well for the faster waves observed in the same region by $\mathrm{Fu}$ and Chelton (2001)].

\section{1) PACIFIC OCEAN}

In the Pacific (White 2000b), the first-mode GS speeds are a reasonable fit to White's data for latitudes of $\pm 14^{\circ}, \pm 18^{\circ}$, and $\pm 22^{\circ}$ but predict a speed significantly larger than found by White at $\pm 10^{\circ}$ (Fig. 17). However, at this latitude (and, indeed, poleward) the second vertical normal mode lies within White's experimental error in both hemispheres. By itself, this is hardly conclusive: there are ever higher modes, and for low speeds it is likely that $a$ mode would fit White's data.

However, White also estimated phase differences between sea surface temperatures and heights, and his contour diagrams of the propagation give some indications of the relative amplitudes of the two signals. In his model, there is a balance between heat lost to the atmosphere and northward advection of heat against the mean $\mathrm{N}-\mathrm{S}$ temperature gradient. This yields

$$
K_{0} T^{\prime}=-\frac{g}{f} \bar{T}_{y} \eta_{x}^{\prime},
$$

where $K_{0}$ is an inverse relaxation time, taken as (one month $)^{-1}, T^{\prime}, \eta^{\prime}$ are perturbation temperatures and sea surface heights respectively, and $\bar{T}_{y}$ is the mean northward surface temperature gradient. White (2000b) argues that this yields the observed $-\pi / 2$ phase shift of temperature as compared with $\eta$, since (6.1) implies

$$
\frac{T^{\prime}}{\eta^{\prime}}=-i k \frac{g}{f} \frac{\bar{T}_{y}}{K_{0}}
$$

The phase shift, however, is not as White predicts at $10^{\circ} \mathrm{S}$. Elsewhere, $\bar{T}_{y}$ is negative (Northern Hemisphere) and positive (Southern Hemisphere). At $10^{\circ} \mathrm{S}$, however, $\bar{T}_{y}$ is positive between $173^{\circ}$ and $199^{\circ} \mathrm{E}$ and between $245^{\circ}$ and $260^{\circ} \mathrm{E}$, changing the sign of the phase difference [this holds both for the World Ocean Atlas data as well as for the map shown by White (2000b), his Fig. 7]. This sign change means that White's model does not fit his data at $10^{\circ} \mathrm{S}$ (a problem shared with the secondmode theory discussed next). The amplitude ratio of $T^{\prime} /$ $\eta^{\prime}$ predicted from (6.2) is also not in good agreement with White's data, predicting ratios of $T^{\prime} / \eta^{\prime}$ of around $0.5^{\circ}$ to $2^{\circ} \mathrm{C} \mathrm{m}^{-1}$ between $\pm 10^{\circ}$ and $\pm 14^{\circ}$ latitude. In contrast, White's anomaly maps and frequency-wavenumber spectra suggest ratios of $10^{\circ}-20^{\circ} \mathrm{C} \mathrm{m}^{-1}$, though with hints of smaller values at $10^{\circ} \mathrm{S}$.

It is also possible to predict the amplitude ratio and phase difference between sea surface temperature and sea surface height from planetary wave theory (appendix C) under straightforward assumptions. With a decay time $\tau$ of about 20 days, both the complete theory using White (2000b)'s estimate of north-south wavenumber of $2500 \mathrm{~km}^{-1}$ poleward, and the simpler theory retaining only east-west advection, predict phase differences for the second mode wave of around -1.4 (i.e., slightly under $\pi / 2$ in amplitude) except at $10^{\circ} \mathrm{S}$ when again the sign change in $\bar{T}_{y}$ causes difficulties. The amplitude ratio is, as in White's theory, somewhat small, ranging from $4^{\circ}$ at $\pm 22^{\circ}$ to $1^{\circ}$ at $\pm 10^{\circ}$. (The amplitude ratio discrepancy deteriorates if the first mode is used because of larger speeds in the denominator.)

We conclude that while second-mode uncoupled planetary wave propagation is not a perfect fit to White's (2000b) data, it does succeed in explaining many of the observed features. ${ }^{7}$ Its failures appear in White's coupled theory also. However, his data clearly demonstrate atmospheric wave propagation also, but whether this is intrinsically coupled (as in White's theory) or merely slaved to oceanic propagation through surface heat fluxes (implicit in ocean-only theory) is unclear.

\footnotetext{
${ }^{7}$ It is not clear whether one would maintain the second mode over the full latitude range $22^{\circ}-10^{\circ}$ or permit the mode to change to the first mode for more poleward latitudes. In the latter case, incoherencies would be evident over the full latitude range.
} 


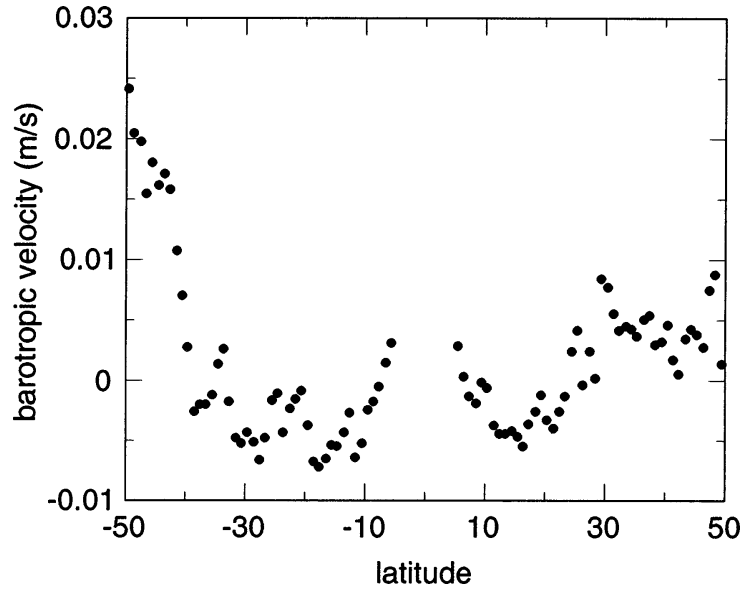

FIG. 18. Zonally averaged barotropic east-west mean velocity, from the OCCAM model.

\section{2) INDIAN OCEAN}

In similar latitude ranges in the South Indian Ocean $\left(10^{\circ}-26^{\circ} \mathrm{S}\right)$, White (2000a) also finds longer-period, slowly propagating waves, with speeds of order $0.05 \mathrm{~m}$ $\mathrm{s}^{-1}$. These, as he indicates, are clearly not first mode planetary waves. They also are clearly unconnected with the planetary waves found by $\mathrm{Fu}$ and Chelton (2001), who report much greater speeds, of $0.13 \mathrm{~m} \mathrm{~s}^{-1}$ at $13^{\circ} \mathrm{S}$. The gradual decay of the waves poleward does not fit any obvious free wave mode (the fourth mode has a speed of $-0.067 \mathrm{~m} \mathrm{~s}^{-1}$ averaged across the same longitude band as White's data at $10^{\circ} \mathrm{S}$, but has a speed lower than $0.01 \mathrm{~m} \mathrm{~s}^{-1}$ at latitudes poleward of $18^{\circ} \mathrm{S}$, which White's data do not show). Little can be drawn from the phase variation, which seems variable; White does not compute it for the Indian Ocean.

This mode, then, would certainly appear to be a coupled mode of some kind, although White's (2000a) model [which is quite different from the White (2000b) model] is not successful at reproducing his observed phase speeds (cf. his Fig. 8).

\section{Effects of mean barotropic flow}

The effects of including the mean barotropic flow are a simple Doppler shift of the waves. We have argued that at low and medium latitudes the barotropic flow is likely to make only a small contribution to the net wave speed, though at high latitudes-especially in the Southern Ocean-this will not hold. Hughes (1995), for example, shows areas in the Antarctic Circumpolar Current where eastward speeds of $0.01-0.02 \mathrm{~m} \mathrm{~s}^{-1}$ are observed, though these are farther south than the $50^{\circ}$ cutoff latitude employed here. While barotropic flows from ocean models are indeed small in most locations, those from fine-resolution eddy-permitting models are somewhat noisy, even when long-term and/or spatially averaged. As an example, we use data from the $1 / 4^{\circ} \mathrm{OC}$ -

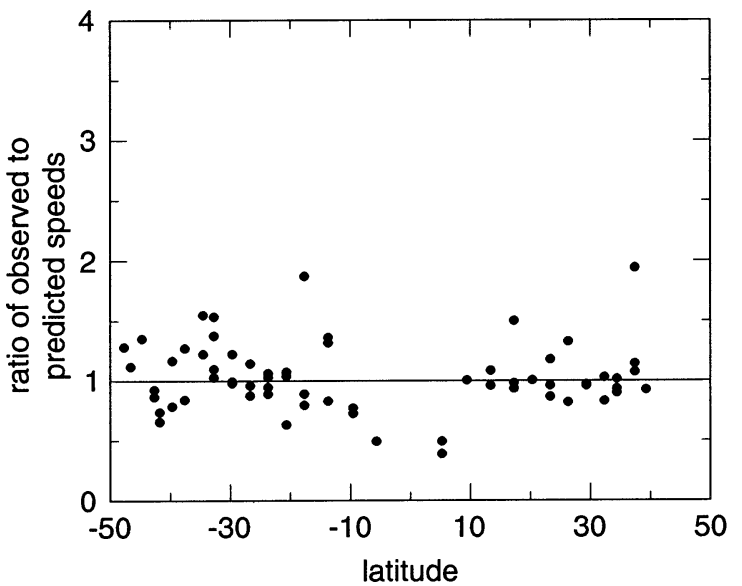

FIG. 19. Ratio of Fu and Chelton (2001) observed speeds to GS theory speeds including a Doppler shift from the OCCAM east-west velocity.

CAM model (Saunders et al. 1999), averaged over three years (whether spatially averaged onto $1^{\circ}$ squares or not makes little difference). At midlatitudes the north-south flow is weak (under $0.002 \mathrm{~m} \mathrm{~s}^{-1}$ almost everywhere). The east-west flow tends to increase in magnitude westward in each gyre as simple Sverdrup theory would predict, reaching as much as $0.01 \mathrm{~m} \mathrm{~s}^{-1}$ in some restricted latitude bands. These values are exceeded in the Antarctic Circumpolar Current. A quantification of the likely effects is given in Fig. 18. The analysis was repeated with Parallel Ocean Program (POP) time-mean barotropic flows, giving almost identical results.

Figure 19 shows the comparison with the Fu and Chelton (2001) data when the barotropic Doppler shift is included. The results are clearly very similar to the purely baroclinic results of Fig. 15, with, as predicted, slight variations at high latitudes. Differences due to the barotropic flow are more clearly visible in Fig. 20, which compares Cipollini's (2001, personal communication) data with the GS theory including the barotropic Doppler effect. At high latitudes there has been a noticeable decrease in the GS speed produced by the Doppler shift, which makes the theory fit the data much less well. (The decrease is not as much as the $0.02 \mathrm{~m} \mathrm{~s}^{-1}$ averaged value, because the number of locations where the GS theory can be evaluated at high latitudes is fairly small. It appears that in such locations, the OCCAM barotropic velocity is weak. In no latitude band did the velocity become eastward.)

Over the majority of the ocean, then, the barotropic flow has little impact on the results presented here, though high-latitude effects are not negligible. ${ }^{8}$

\footnotetext{
${ }^{8}$ No ray calculations have been made retaining the barotropic mean flow, and it is possible that its inclusion might modify the regions of convergence and divergence.
} 


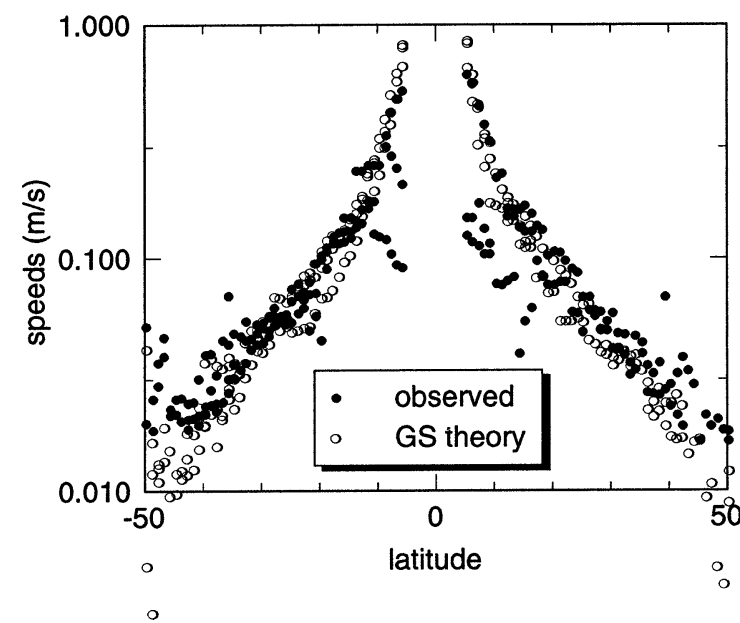

FIG. 20. Cipollini's observed altimeter-derived speeds and the GS theory including the OCCAM barotropic velocity.

\section{Discussion}

The results of KBI presented group velocities averaged over many orientations of the wavevector. This paper has examined two questions. First, whether this approach gave a "correct" representation, by comparing the KBI results with those from ray theory, where the rays initiate from an eastern boundary. We find that, with a few exceptions due to differing averaging areas and smoothing, that ray-based and local theory give essentially the same answers, so that results tabulated from local calculations, even using less-smoothed data, can be relied upon for predictions of planetary wave propagation.

The second question was whether the wave properties predicted by our solutions can be observed remotely in sea surface height measurements. Killworth et al. (1997), who included baroclinic mean flow, had already found a generic speedup of normal modes over standard theory for a resting ocean. KBI, including topographic slopes as well, found a second speedup, the physical reason for which remain unclear. Comparison of our group velocity estimates with radon transform estimates of phase speed in sea surface height data shows very good agreement, with the remaining scatter of the same size as the scatter between estimates of observed wave speed using different filtering algorithms. However, predicted surface amplitudes for waves emanating from an eastern boundary do not agree well with observed amplitudes.

It would be useful to relax certain of the assumptions made in this theory. It is possible to include non-longwave effects (KB99 give a method in the case of topographic slopes but no mean flow) though the algebra, already tedious, would become excessive. The predominant difficulty remains the WKBJ requirement that the mean flow and topography should vary only slowly; clearly this is not the case in general. This raises issues such as scattering, the effects of gaps and sills, random topography, and so on, all of which are considerable problems in their own right, not to mention atmospheric coupling. Nonetheless, the excellent comparison between our results and observations give us cause to believe that this theory gives a useful first-order approximation to the propagation of long planetary waves.

Acknowledgments. We repeat our thanks to the SOC satellite team for their encouragement and collaboration, and to the referees for their insight and for noting an initial error in the formulation for the surface amplitude behavior. Anita Grezio and Vladimir Ivchenko provided the POP data. This work is part of the James Rennell Division Care Project CSP1.

\section{APPENDIX A}

\section{Second Derivatives of the Dispersion Relation}

Section 3 of KBI, and its appendices, have demonstrated how the eigenvalue problem can be solved numerically, and the first derivative of the dispersion relation found from simple vertical integrals. In order to detect possible caustics in the ray tracing in this paper, second derivatives of the dispersion relation are also required. We demonstrate here that these too can be obtained numerically.

We begin by assuming that (2.2) has been solved numerically for $F$ and that $\omega_{X}$ has been computed as in $\mathrm{KBI}$, where $X$ is one of $(k, l, \lambda, \theta)$. If the latter is known, then from the derivative of (2.2) w.r.t. $X$, namely

$$
L\left(F_{X}\right)+L_{X}(F)+\omega_{X} L_{\omega}(F)=0,
$$

we can solve for $F_{X}$, which can be tabulated at the same depths as $F$ has been computed. Differentiating (A.1) w.r.t. $Y$ (again one of $\lambda, \theta, k, l$ ), we obtain

$$
\begin{aligned}
& L\left(F_{X Y}\right)+L_{Y}\left(F_{X}\right)+\omega_{Y} L_{\omega}\left(F_{X}\right)+L_{X Y}(F)+L_{X}\left(F_{Y}\right) \\
& \quad+\omega_{Y} L_{X \omega}(F)+\omega_{X Y} L_{\omega}(F)+\omega_{X} L_{\omega}\left(F_{Y}\right) \\
& \quad+\omega_{X} L_{Y \omega}(F)+\omega_{X} \omega_{Y} L_{\omega \omega}(F)=0 .
\end{aligned}
$$

This has boundary conditions

$$
\begin{gathered}
F_{X Y}=F_{X Y z}=0, \quad z=0 . \\
F_{X Y}(-H)+\alpha F_{X Y z}(-H)-H_{Y} F_{X z}(-H)+\alpha_{Y} F_{X z}(-H) \\
-\alpha H_{Y} F_{X z z}(-H)-H_{X Y} F_{z}(-H)-H_{X} F_{Y z}(-H) \\
+H_{X} H_{Y} F_{z z}(-H)+\alpha_{X Y} F_{z}(-H)+\alpha_{X} F_{Y z}(-H) \\
-\alpha_{X} H_{Y} F_{z z}(-H)-\alpha_{Y} H_{X} F_{z z}(-H)-\alpha H_{X Y} F_{z z}(-H) \\
-\alpha H_{X} F_{Y z z}(-H)+\alpha H_{X} H_{Y} F_{z z z}(-H)=0 .
\end{gathered}
$$

Cross-multiply (A.2) by $F$ minus (2.2) times $F_{X Y}$ and integrate top to bottom to eliminate $F_{X Y}$ : 


$$
\begin{aligned}
\left(F \frac{F_{X Y z}}{R}-F_{X Y} \frac{F_{z}}{R}\right)_{-H}^{0} & \\
+\int_{-H}^{0} d z\{F[ & L_{Y}\left(F_{X}\right)+\omega_{Y} L_{\omega}\left(F_{X}\right)+L_{X Y}(F)+L_{X}\left(F_{Y}\right) \\
& +\omega_{Y} L_{X \omega}(F)+\omega_{X Y} L_{\omega}(F)+\omega_{X} L_{\omega}\left(F_{Y}\right) \\
& \left.\left.+\omega_{X} L_{Y \omega}(F)+\omega_{X} \omega_{Y} L_{\omega \omega}(F)\right]\right\}=0 .
\end{aligned}
$$

The surface values vanish, leaving

$$
\begin{aligned}
& \frac{F_{z}(-H)}{R(-H)}\left[F_{X Y}(-H)+\alpha F_{X Y}(-H)\right] \\
& +\int_{-H}^{0} d z\left\{F \left[L_{Y}\left(F_{X}\right)+\omega_{Y} L_{\omega}\left(F_{X}\right)+L_{X Y}(F)+L_{X}\left(F_{Y}\right)\right.\right. \\
& +\omega_{Y} L_{X \omega}(F)+\omega_{X Y} L_{\omega}(F)+\omega_{X} L_{\omega}\left(F_{Y}\right) \\
& \left.\left.+\omega_{X} L_{Y \omega}(F)+\omega_{X} \omega_{Y} L_{\omega \omega}(F)\right]\right\}=0 .
\end{aligned}
$$

This becomes, using the bottom boundary condition, the lengthy expression

$$
\begin{gathered}
-\frac{F_{z}(-H)}{R(-H)}\left[-H_{Y} F_{X z}(-H)+\alpha_{Y} F_{X z}(-H)-\alpha H_{Y} F_{X z z}(-H)-H_{X Y} F_{z}(-H)-H_{X} F_{Y z}(-H)+H_{X} H_{Y} F_{z z}(-H)\right. \\
+\alpha_{X Y} F_{z}(-H)+\alpha_{X} F_{Y z}(-H)-\alpha_{X} H_{Y} F_{z z}(-H)-\alpha_{Y} H_{X} F_{z z}(-H)-\alpha H_{X Y} F_{z z}(-H)-\alpha H_{X} F_{Y z z}(-H) \\
\left.\quad+\alpha H_{X} H_{Y} F_{z z z}(-H)\right] \\
+\int_{-H}^{0} d z\left\{F \left[L_{Y}\left(F_{X}\right)+\omega_{Y} L_{\omega}\left(F_{X}\right)+L_{X Y}(F)+L_{X}\left(F_{Y}\right)+\omega_{Y} L_{X \omega}(F)+\omega_{X Y} L_{\omega}(F)+\omega_{X} L_{\omega}\left(F_{Y}\right)+\omega_{X} L_{Y \omega}(F)\right.\right. \\
\left.\left.\quad+\omega_{X} \omega_{Y} L_{\omega \omega}(F)\right]\right\}=0 .
\end{gathered}
$$

Note that this contains $F_{X z z}(-H)$ and $F_{X z}(-H)$, which are evaluated below. The $\omega_{X Y}$ term involves the same integral as already computed for the first derivative in KBI.

All the integral terms can be computed, albeit tediously. In sequence, they are

$$
\begin{array}{rl}
\int_{-H}^{0} & F L_{Y}\left(F_{X}\right) d z \\
= & \int_{-H}^{0} F\left[\frac{\partial}{\partial z}\left(\frac{-R_{Y}}{R^{2}} F_{X z}\right)+\left(\frac{S}{R^{2}}\right)_{Y} F_{X}\right] d z \\
= & F(-H) \frac{R_{Y}(-H)}{R^{2}(-H)} F_{X z}(-H) \\
& +\int_{-H}^{0}\left[\frac{R_{Y}}{R^{2}} F_{z} F_{X z}+\left(\frac{S}{R^{2}}\right)_{Y} F F_{X}\right] d z \\
\int_{-H}^{0} & F L_{\omega}\left(F_{X}\right) d z \\
= & \int_{-H}^{0} F\left[\frac{\partial}{\partial z}\left(\frac{F_{X z}}{R^{2}}\right)+\frac{2 S}{R^{3}} F_{X}\right] d z
\end{array}
$$

The next set need definitions of derivatives of $L$ :

$$
\begin{aligned}
L_{X Y}(F) & =\frac{\partial}{\partial z}\left(-\frac{R_{X Y}}{R^{2}} F_{z}+2 \frac{R_{X} R_{Y}}{R^{3}} F_{z}\right)+\left(\frac{S}{R^{2}}\right)_{X Y} F \\
L_{X \omega}(F) & =\frac{\partial}{\partial z}\left(-\frac{R_{X \omega}}{R^{2}} F_{z}+2 \frac{R_{X} R_{\omega}}{R^{3}} F_{z}\right)+\left(\frac{S_{\omega}}{R^{2}}-2 \frac{S R_{\omega}}{R^{3}}\right)_{X} F \\
& =\frac{\partial}{\partial z}\left(-2 \frac{R_{X} F_{z}}{R^{3}}\right)+\left(\frac{2 S}{R^{3}}\right)_{X} F \\
L_{\omega \omega}(F) & =\frac{\partial}{\partial z}\left(2 \frac{F_{z}}{R^{3}}\right)+\frac{6 S}{R^{4}} F .
\end{aligned}
$$

Then, continuing,

$$
\begin{aligned}
\int_{-H}^{0} F L_{X Y}(F) d z= & -F(-H)\left[-\frac{R_{X Y}(-H)}{R^{2}(-H)} F_{z}(-H)+2 \frac{R_{X}(-H) R_{Y}(-H)}{R^{3}(-H)} F_{z}(-H)\right] \\
& +\int_{-H}^{0}\left[-F_{z}^{2}\left(-\frac{R_{X Y}}{R^{2}}+2 \frac{R_{X} R_{Y}}{R^{3}}\right)+\left(\frac{S}{R^{2}}\right)_{X Y} F^{2}\right] d z
\end{aligned}
$$




$$
\begin{aligned}
& \int_{-H}^{0} F L_{X \omega}(F) d z=2 F(-H) \frac{R_{X}(-H) F_{z}(-H)}{R^{3}(-H)}+\int_{-H}^{0}\left[2 \frac{R_{X} F_{z}^{2}}{R^{3}}+\left(\frac{2 S}{R^{3}}\right)_{X} F^{2}\right] d z \\
& \int_{-H}^{0} F L_{\omega \omega}(F) d z=-2 F(-H) \frac{F_{z}(-H)}{R^{3}(-H)}+\int_{-H}^{0}\left(-2 \frac{F_{z}^{2}}{R^{3}}+\frac{6 S F^{2}}{R^{4}}\right) d z .
\end{aligned}
$$

Substitution of (A.8)-(A.15) into (A.7) yields $\omega_{X Y}$ as required.

\section{APPENDIX B}

\section{An Evolution Equation for the Wave Amplitude}

To understand the evolution of the amplitude, we explicitly seek a WKBJ formulation of the entire problem. First, we need to derive alternative expressions for group velocity. Consider

$$
\tilde{L}(F) \equiv R^{2} L(F) \equiv R F_{z z}-R_{z} F_{z}+S F=0 .
$$

Taking $\partial / \partial X$ of this gives

$$
\tilde{L}\left(F_{X}\right)+\tilde{L}_{X}(F)+\omega_{X} \tilde{L}_{\omega}(F)=0 .
$$

Taking $\left(1 / R^{2}\right)\left[F_{X}(\mathrm{~B} .1)-F(\mathrm{~B} .2)\right]$, and integrating top to bottom, we have

$$
\begin{gathered}
\int_{-H}^{0}\left[F_{X} L(F)-F L\left(F_{X}\right)\right] d z-\int_{-H}^{0} \frac{F}{R^{2}} \tilde{L}_{X}(F) d z \\
-\omega_{X} \int_{-H}^{0} \frac{F}{R^{2}} \tilde{L}_{\omega}(F) d z=0 .
\end{gathered}
$$

The first term becomes boundary terms which are precisely as in KBI, and if we restrict $X$ to $k, l$ so that $H_{X}$ $=0$,

$$
\begin{aligned}
& \frac{-F_{z}(-H)}{R(-H)}\left[-\alpha_{X} F_{z}(-H)\right] \\
& \quad=\int_{-H}^{0} \frac{F}{R^{2}} \tilde{L}_{X}(F) d z+\omega_{X} \int_{-H}^{0} \frac{F}{R^{2}} \tilde{L}_{\omega}(F) d z .
\end{aligned}
$$

Now

$$
\begin{aligned}
\tilde{L} & =R^{2} L \Rightarrow \tilde{L}_{X}=R^{2} L_{X}+2 R R_{X} L ; \\
\tilde{L}_{\omega} & =R^{2} L_{\omega}+2 R R_{\omega} L
\end{aligned}
$$

so that

$$
\int_{-H}^{0} \frac{F}{R^{2}} \tilde{L}_{X}(F) d z=\int_{-H}^{0}\left[F L_{X}(F)+2 \frac{R_{X}}{R} F L(F)\right] d z
$$

and the second term vanishes by definition, leaving the balance

$$
\int_{-H}^{0} \frac{F}{R^{2}} \tilde{L}_{X}(F) d z=\int_{-H}^{0} F L_{X}(F) d z,
$$

where here $X$ can include $\omega$. Thus alternative expressions for group velocity are

$$
\begin{aligned}
& \omega_{k} \int_{-H}^{0} F L_{\omega}(F) d z \\
& \quad=\int_{-H}^{0} \frac{F}{R^{2}} \tilde{L}_{k}(F) d z+\frac{F_{z}^{2}(-H)}{R(-H)}\left(-\frac{l \tan \theta}{k^{2}} H_{\lambda}\right)
\end{aligned}
$$

$$
\begin{aligned}
& \omega_{l} \int_{-H}^{0} F L_{\omega}(F) d z \\
& \quad=\int_{-H}^{0} \frac{F}{R^{2}} \tilde{L}_{l}(F) d z+\frac{F_{z}^{2}(-H)}{R(-H)}\left(\frac{\tan \theta}{k} H_{\lambda}\right) .
\end{aligned}
$$

These will be needed below.

To solve the WKBJ problem, we pose

$$
\begin{aligned}
M= & {\left[G_{0}(\lambda, \theta, z)+G_{1}(\lambda, \theta, z)+\cdots\right] } \\
& \times \operatorname{expi}(k \lambda+l \theta-\omega t),
\end{aligned}
$$

where $G_{1} / G_{0}$ is of order the small parameter (wavelength scale/gyre scale); $G$ is used instead of $F$ to remind us that it has a scale, rather than being normalized. The boundary conditions are

$$
G_{0}=G_{1}=0, \quad z=0 \quad \text { and }
$$

$M_{\lambda}+\tan \theta\left(M_{z \lambda} H_{\theta}-M_{z \theta} H_{\lambda}\right)=0, \quad z=-H$,

which becomes

$$
\begin{aligned}
& i k\left(G_{0}+G_{1}\right)+G_{0 \lambda} \\
& +\tan \theta\left[H_{\theta}\left(i k G_{0 z}+i k G_{1 z}+G_{0 z \lambda}\right)\right. \\
& \left.\quad-H_{\lambda}\left(i l G_{0 z}+i l G_{1 z}+G_{0 z \theta}\right)\right]=0, \quad z=-H .
\end{aligned}
$$

Expanding this gives to leading order

$$
\begin{aligned}
G_{0}+\tan \theta G_{0 z}\left(H_{\theta}-\frac{l}{k} H_{\lambda}\right) & \equiv G_{0}+\alpha G_{0 z}=0, \\
z & =-H,
\end{aligned}
$$

and to the next order

$$
\begin{array}{r}
G_{1}+\alpha G_{1 z}+\frac{1}{i k}\left[G_{0 \lambda}+\tan \theta\left(H_{\theta} G_{0 z \lambda}-H_{\lambda} G_{0 z \theta}\right)\right]=0, \\
z=-H . \quad \text { (B.10) }
\end{array}
$$

The equation of motion [KBI:(2.8)] now becomes

$$
\begin{aligned}
& -i \omega\left(G_{0 z z}+G_{1 z z}\right)+\frac{\bar{u}}{a \cos \theta}\left[i k\left(G_{0 z z}+G_{1 z z}\right)+G_{0 z z \lambda}\right] \\
& +\frac{\bar{v}}{a}\left[i l\left(G_{0 z z}+G_{1 z z}\right)+G_{0 z z \theta}\right]
\end{aligned}
$$




$$
\begin{aligned}
& -\frac{\bar{u}_{z}}{a \cos \theta}\left[i k\left(G_{0 z}+G_{1 z}\right)+G_{0 z \lambda}\right] \\
& -\frac{\bar{v}_{z}}{a}\left[i l\left(G_{0 z}+G_{1 z}\right)+G_{0 z \theta}\right] \\
& +\frac{N^{2}}{f a^{2} \sin \theta}\left[i k\left(G_{0}+G_{1}\right)+G_{0 \lambda}\right]=0 .
\end{aligned}
$$

Thus

$$
R^{2} L\left(G_{0}\right)=0
$$

to leading order, and

$$
\begin{aligned}
R^{2} L\left(G_{1}\right)-i( & \frac{\bar{u}}{a \cos \theta} G_{0 z z \lambda}+\frac{\bar{v}}{a} G_{0 z z \theta}-\frac{\bar{u}_{z}}{a \cos \theta} G_{0 z \lambda} \\
& \left.-\frac{\bar{v}_{z}}{a} G_{0 z \theta}+\frac{N^{2}}{f a^{2} \sin \theta} G_{0 \lambda}\right)=0 .
\end{aligned}
$$

$G_{0}$ is some (unknown) multiple of the eigensolution $F$. We shall write

$$
G_{0}=a_{0} F,
$$

where the behavior of $a_{0}(\lambda, \theta)$, the amplitude of the solution, is to be found.

Now integrate $R^{-2}\left[(\mathrm{~B} .11) G_{1}-(\mathrm{B} .12) G_{0}\right]$ and integrate from top to bottom:

Equation (B.11) is merely the eigenvalue problem, so The lhs of (B.14) becomes

$$
\begin{aligned}
\left(\frac{G_{1} G_{0 z}-G_{0} G_{1 z}}{R}\right)_{-H}^{0} & =-\frac{1}{R(-H)}\left\langle G_{1}\left(-\frac{G_{0}}{\alpha}\right)-G_{0}\left\{-\frac{G_{1}}{\alpha}+\frac{i}{\alpha k}\left[G_{0 \lambda}+\tan \theta\left(H_{\theta} G_{0 z \lambda}-H_{\lambda} G_{0 z \theta}\right)\right]\right\}\right\rangle \\
& =\frac{i}{\alpha k R(-H)} G_{0}(-H)\left[G_{0 \lambda}+\tan \theta\left(H_{\theta} G_{0 z \lambda}-H_{\lambda} G_{0 z \theta}\right)\right]_{z=-H} .
\end{aligned}
$$

The rhs of (B.14) becomes

$$
-i \int_{-H}^{0} \frac{G_{0}}{R^{2}}\left[\tilde{L}_{k}\left(G_{0 \lambda}\right)+\tilde{L}_{l}\left(G_{0 \theta}\right)\right] d z
$$

so that (B.14) can be written

$$
\frac{1}{\alpha k R(-H)} G_{0}(-H)\left[G_{0 \lambda}+\tan \theta\left(H_{\theta} G_{0 z \lambda}-H_{\lambda} G_{0 z \theta}\right)\right]_{z=-H}=-\int_{-H}^{0} \frac{G_{0}}{R^{2}}\left[\tilde{L}_{k}\left(G_{0 \lambda}\right)+\tilde{L}_{l}\left(G_{0 \theta}\right)\right] d z .
$$

Since $G_{0}=a_{0} F$ (the latter of which is known), $G_{0 \lambda}=a_{0 \lambda} F+a_{0} F_{\lambda}$ (and again $F, F_{\lambda}$ are known), and similarly for the $\theta$ derivatives, substitution into (B.17) yields, cancelling a factor of $-a_{0}$,

$$
\begin{gathered}
-\frac{F_{z}(-H)}{k R(-H)}\left\{a_{0 \lambda} F+a_{0} F_{\lambda}+\tan \theta\left[H_{\theta}\left(a_{0 \lambda} F_{z}+a_{0} F_{z \lambda}\right)-H_{\lambda}\left(a_{0 \theta} F_{z}+a_{0} F_{z \theta}\right)\right]\right\}_{z=-H} \\
=\int_{-H}^{0} \frac{F}{R^{2}}\left[a_{0 \lambda} \tilde{L}_{k}(F)+a_{0} \tilde{L}_{k}\left(F_{\lambda}\right)+a_{0 \theta} \tilde{L}_{l}(F)+a_{0} \tilde{L}_{l}\left(F_{\theta}\right)\right] d z .
\end{gathered}
$$

Collecting terms in $a_{0}$, we have

$$
\begin{aligned}
& a_{0 \lambda}\left\{\int_{-H}^{0} \frac{F}{R^{2}} \tilde{L}_{k}(F) d z-\frac{F_{z}(-H)}{k R(-H)}\left[F(-H)+\tan \theta H_{\theta} F_{z}(-H)\right]\right\}+a_{0 \theta}\left\{\int_{-H}^{0} \frac{F}{R^{2}} \tilde{L}_{l}(F) d z-\frac{F_{z}(-H)}{k R(-H)}\left[-\tan \theta H_{\lambda} F_{z}(-H)\right]\right\} \\
& \quad+a_{0}\left\langle\int_{-H}^{0} \frac{F}{R^{2}}\left[\tilde{L}_{k}\left(F_{\lambda}\right)+\tilde{L}_{l}\left(F_{\theta}\right)\right] d z-\frac{F_{z}(-H)}{k R(-H)}\left\{F_{\lambda}(-H)+\tan \theta\left[H_{\theta} F_{z \lambda}(-H)-H_{\lambda} F_{z \theta}(-H)\right]\right\}\right\rangle=0 .
\end{aligned}
$$

Now in the first term, we can use the bottom condition to simplify it:

$$
F(-H)+\tan \theta H_{\theta} F_{z}(-H)=\frac{l}{k} \tan \theta H_{\lambda} F_{z}(-H)
$$

so that (B.19) becomes 


$$
\begin{aligned}
& a_{0 \lambda}\left[\int_{-H}^{0} \frac{F}{R^{2}} \tilde{L}_{k}(F) d z-\frac{l F_{z}^{2}(-H)}{k^{2} R(-H)} \tan \theta H_{\lambda}\right]+a_{0 \theta}\left[\int_{-H}^{0} \frac{F}{R^{2}} \tilde{L}_{l}(F) d z+\frac{F_{z}^{2}(-H)}{k R(-H)} \tan \theta H_{\lambda}\right] \\
& \quad+a_{0}\left\langle\int_{-H}^{0} \frac{F}{R^{2}}\left[\tilde{L}_{k}\left(F_{\lambda}\right)+\tilde{L}_{l}\left(F_{\theta}\right)\right] d z-\frac{F_{z}(-H)}{k R(-H)}\left\{F_{\lambda}(-H)+\tan \theta\left[H_{\theta} F_{z \lambda}(-H)-H_{\lambda} F_{z \theta}(-H)\right]\right\}\right\rangle=0 .
\end{aligned}
$$

Equation (B.13) can be rewritten, using (B.7a) and (B.7b), as

$$
\left[\int_{-H}^{0} F L_{\omega}(F) d z\right] \frac{D a_{0}}{D s}=a_{0}\left\langle\int_{-H}^{0} \frac{F}{R^{2}}\left[\tilde{L}_{k}\left(F_{\lambda}\right)+\tilde{L}_{l}\left(F_{\theta}\right)\right] d z-\frac{F_{z}(-H)}{k R(-H)}\left\{F_{\lambda}(-H)+\tan \theta\left[H_{\theta} F_{z \lambda}(-H)-H_{\lambda} F_{z \theta}(-H)\right]\right\}\right\rangle .
$$

Equation (B.21) is the required evolution equation for the amplitude. With the exception of the first two integrals on the rhs, all terms in the various integral and boundary functions are already known. The two additional integrals can be computed by using (B.5) on $\tilde{L}_{k}$, $\tilde{L}_{l}$ and Eq. (3.1) in KBI to convert the awkward integrals. Thus (B.21) can be followed along a ray.

\section{APPENDIX C}

\section{Sea Surface Temperature within Planetary Waves}

Sea surface temperature is an active tracer, and so we consider the perturbation density equation:

$$
\rho_{t}^{\prime}+\bar{u} \rho_{x}^{\prime}+u^{\prime} \bar{\rho}_{x}+\bar{v} \rho_{y}^{\prime}+v^{\prime} \bar{\rho}_{y}=-\frac{\rho^{\prime}}{\tau} .
$$

Here quantities are evaluated at the surface, and $\tau$ is a relaxation time. (Ocean-atmosphere fluxes of heat and moisture are not necessarily connected, but a simple decay term has been used by many authors for simplicity. A more complicated solution would be to treat heat and salt separately ab initio.) Setting

$$
\boldsymbol{\nabla} \bar{\rho}=\rho_{0}(-\alpha \nabla \bar{T}+\beta \nabla \bar{S}),
$$

where $\alpha$ and $\beta$ are expansion coefficients for heat and salt and assuming by necessity that perturbation density and temperature are related by

$$
\rho^{\prime}=-\rho_{0} \alpha T^{\prime}
$$

since salinity is not yet remotely observable, (B.1) becomes

$$
\begin{gathered}
T_{t}^{\prime}+\bar{u} T_{x}^{\prime}+\bar{v} T_{y}^{\prime}+u^{\prime}\left(\bar{T}_{x}-\frac{\beta}{\alpha} \bar{S}_{x}\right) \\
+v^{\prime}\left(\bar{T}_{y}-\frac{\beta}{\alpha} \bar{S}_{y}\right)=-\frac{T^{\prime}}{\tau} .
\end{gathered}
$$

We assume that $u^{\prime}, v^{\prime}$ are related by geostrophy to the surface height perturbation $\eta^{\prime}$ by

$$
u^{\prime}=-\frac{g}{f} \eta_{y}^{\prime}, \quad v^{\prime}=\frac{g}{f} \eta_{x}^{\prime}
$$

and that $T^{\prime}, \eta^{\prime}$ are of the form

$$
T^{\prime}=T_{0} e^{i \phi}, \eta^{\prime}=\eta_{0} e^{i \phi}, \phi=k \lambda+l \theta-\omega t .
$$

Then

$$
\begin{aligned}
& T_{0}\left(\frac{\bar{u} k}{a \cos \theta}+\frac{\bar{v} l}{a}-\omega-\frac{i}{\tau}\right) \\
& \quad+\frac{g \eta_{0}}{f}\left(\frac{k \hat{T}_{y}}{a}-\frac{l \hat{T}_{x}}{a \cos \theta}\right)=0,
\end{aligned}
$$

where

$$
\nabla \hat{T}=\nabla \bar{T}-\frac{\beta}{\alpha} \nabla \bar{S}
$$

is an "effective temperature gradient." (In many areas of the ocean this is dominated by the actual temperature gradient.)

Replacing the angular wavevector with its more traditional inverse distance counterpart $\left(k^{\prime}=k / a \cos \theta, l^{\prime}\right.$ $=(/ a)$ gives

$$
\frac{T_{0}}{\eta_{0}}=-\frac{g}{f} \frac{\left(k^{\prime} \hat{T}_{y}-l^{\prime} \hat{T}_{x}\right)}{\left(\bar{u} k^{\prime}+\bar{v} l^{\prime}-\omega-i / \tau\right)},
$$

and, if we use (4.4) in KBI,

$$
\omega=k^{\prime} c^{\mathrm{gx}}+l^{\prime} c^{\mathrm{gy}},
$$

to substitute for $\omega$ in the denominator, we get

$$
\frac{T_{0}}{\eta_{0}}=-\frac{g}{f} \frac{\left(k^{\prime} \hat{T}_{y}-l^{\prime} \hat{T}_{x}\right)}{\left[k^{\prime}\left(\bar{u}-c^{\mathrm{gx}}\right)+l^{\prime}\left(\bar{v}-c^{\mathrm{gy}}\right)-i / \tau\right]},
$$

which gives both amplitude and phase of the temperature signal compared with the sea surface height signal if $k^{\prime}, l^{\prime}$ are known. In the equatorial regions discussed in the paper, the terms in $l^{\prime}$ are small and the system reduces to the simpler

$$
\begin{aligned}
& \frac{T_{0}}{\eta_{0}}=-\frac{g}{f} \frac{k^{\prime} \hat{T}_{y}}{\left[k^{\prime}\left(\bar{u}-c^{g x}\right)-i / \tau\right]} \quad \text { or } \\
& \frac{T_{0}}{\eta_{0}}=-\frac{i k^{\prime} g}{f} \hat{T}_{y} \frac{\tau}{1-i \tilde{\omega} \tau},
\end{aligned}
$$


where $\tilde{\omega}=\omega-k^{\prime} \bar{u}=\omega\left(1-\bar{u} / c^{\mathrm{gx}}\right)$ is a frequency Doppler-shifted by the surface flow. In this case, using $\omega=k^{\prime} c^{\mathrm{gx}}$, the wavenumber $k^{\prime}$ (or $k$ ) can be estimated for a given frequency.

For small $\tilde{\omega} \tau$ (usually approximately the case), the phase of $T^{\prime}$ as compared with $\eta^{\prime}$ becomes $(\pi / 2) \operatorname{sgn}\left(\hat{T}_{y}\right)$ $\operatorname{sgn}(f)$. For large $\tilde{\omega} \tau$, the phase becomes $\pi$ (Northern Hemisphere, $\hat{T}_{y}$ positive; Southern Hemisphere, $\hat{T}_{y}$ negative) or zero ( $\hat{T}_{y}$ taking opposite signs). The amplitude ratio increases monotonically with $\tilde{\omega} \tau$ to an asymptotic maximum.

\section{REFERENCES}

Anderson, D. L. T., and P. D. Killworth, 1997: Spin-up of a stratified ocean with topography. Deep-Sea Res., 24, 709-732.

Antonov, J., S. Levitus, T. P. Boyer, M. Conkright, T. O'Brien, and C. Stephens, 1998: Temperature of the Atlantic/Pacific/Indian Ocean. Vols. 1-3, World Ocean Atlas 1998, NOAA Atlas NESDIS 27, $166 \mathrm{pp}$.

Barnier, B., 1988: A numerical study of the influence of the MidAtlantic Ridge on nonlinear first-mode baroclinic Rossby waves generated by seasonal winds. J. Phys. Oceanogr., 18, 417-433.

Boyer, T. P., S. Levitus, J. Antonov, M. Conkright, T. O'Brien, and C. Stephens, 1998: Salinity of the Atlantic/Pacific/Indian Ocean. Vols. 4-6, World Ocean Atlas 1998, NOAA Atlas NESDIS 30 $166 \mathrm{pp}$.

Chelton, D. B., and M. G. Schlax, 1996: Global observations of Oceanic Rossby Waves. Science, 272, 234-238.

Cipollini, P., D. Cromwell, M. S. Jones, G. D. Quartly, and P. G. Challenor, 1997: Concurrent altimeter and infrared observations of Rossby wave propagation near $34^{\circ} \mathrm{N}$ in the Northeast Atlantic. Geophys. Res. Lett., 24, 889-892.

Fu, L. L., and D. B. Chelton, 2001: Large-scale ocean circulation. Satellite Altimetry and Earth Sciences, L. L. Fu and A. Cazenave, Eds., Academic Press, 133-169.

Hallberg, R., 1997: Localized coupling between surface and bottomintensified flow over topography. J. Phys. Oceanogr., 27, 977998.

Hill, K. L., I. S. Robinson, and P. Cipollini, 2000: Propagation characteristics of extratropical planetary waves observed in the
ATSR global sea surface temperature record. J. Geophys. Res., 105, 21 927-21945.

Hughes, C. W., 1995: Rossby waves in the Southern Ocean: A comparison of TOPEX/POSEIDON altimetry with model predictions. J. Geophys. Res., 100, 15 933-15950.

Killworth, P. D., and J. R. Blundell, 1999: The effect of bottom topography on the speed of long extra-tropical planetary waves. J. Phys. Oceanogr., 29, 2689-2710.

—_, and _ 2003: Long extratropical planetary wave propagation in the presence of slowly varying mean flow and bottom topography. Part I: The local problem. J. Phys. Oceanogr., 33, 784801.

- D. B. Chelton, and R. A. de Szoeke, 1997: The speed of observed and theoretical long extratropical planetary waves. $J$. Phys. Oceanogr., 27, 1946-1966.

Lighthill, M. J., 1978: Waves in Fluids. Cambridge University Press, $504 \mathrm{pp}$.

Liu, Z., 1999: Forced planetary wave response in a thermocline gyre. J. Phys. Oceanogr., 29, 1036-1055.

National Geophysical Data Center, 1988: Data Announcement 88MG-02: Digital Relief of the Surface of the Earth. NOAA National Geophysics Data Center, CD-ROM. [Available from NOAA, National Geophysical Data Center, Boulder, CO 803053328.]

Numerical Algorithms Group Ltd., cited 1999: NAG Fortran Library Manual, Mark 19. [Available online at http://www.nag.co.uk/ numeric/fl/manual19/html/mark19.html.]

Saunders, P. M., A. C. Coward, and B. A. de Cuevas, 1999: Circulation of the Pacific Ocean seen in a global ocean model (OCCAM). J. Geophys. Res., 104, 18 281-18 299.

Schopf, P., D. L. T. Anderson, and R. Smith, 1981: Beta-dispersion of low frequency Rossby waves. Dyn. Atmos. Oceans, 5, 187214.

Tailleux, R., and J. C. McWilliams, 2000: Acceleration, creation, and depletion of wind-driven, baroclinic Rossby waves over an ocean ridge. J. Phys. Oceanogr., 30, 2186-2213.

Welander, P., 1959: An advective model of the ocean thermocline. Tellus, 11, 309-318

White, W. B., 2000a: Coupled Rossby waves in the Indian Ocean on interannual timescales. J. Phys. Oceanogr., 30, 2972-2988.

_- 2000b: Tropical coupled Rossby waves in the Pacific oceanatmosphere system. J. Phys. Oceanogr., 30, 1245-1264.

Yang, H., 2000: Evolution of long planetary wave packets in a continuously stratified ocean. J. Phys. Oceanogr., 30, 2111-2123. 